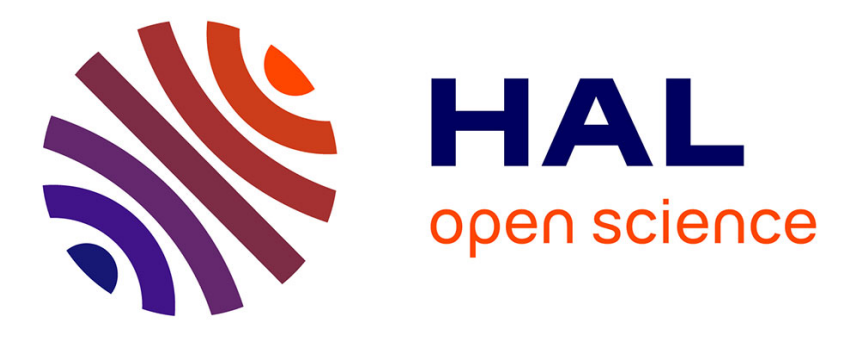

\title{
Adapting Consistency in Constraint Solving
}

Amine Balafrej, Christian Bessiere, Anastasia Paparrizou, Gilles Trombettoni

\section{To cite this version:}

Amine Balafrej, Christian Bessiere, Anastasia Paparrizou, Gilles Trombettoni. Adapting Consistency in Constraint Solving. Christian Bessiere; Luc De Raedt; Lars Kotthoff; Siegfried Nijssen; Barry O'Sullivan; Dino Pedreschi. Data Mining and Constraint Programming - Foundations of a CrossDisciplinary Approach, 10101, Springer, pp.226-253, 2016, Lecture Notes in Computer Science, 9783-319-50136-9. 10.1007/978-3-319-50137-6_9. hal-01711595

\section{HAL Id: hal-01711595 \\ https://hal.science/hal-01711595}

Submitted on 19 Feb 2018

HAL is a multi-disciplinary open access archive for the deposit and dissemination of scientific research documents, whether they are published or not. The documents may come from teaching and research institutions in France or abroad, or from public or private research centers.
L'archive ouverte pluridisciplinaire HAL, est destinée au dépôt et à la diffusion de documents scientifiques de niveau recherche, publiés ou non, émanant des établissements d'enseignement et de recherche français ou étrangers, des laboratoires publics ou privés. 


\title{
Adapting Consistency in Constraint Solving*
}

\author{
Amine Balafrej ${ }^{1}$ \\ Christian Bessiere ${ }^{2}$ \\ Anastasia Paparrizou $^{2} \quad$ Gilles Trombettoni ${ }^{2}$ \\ ${ }^{1}$ TASC (INRIA/CNRS), Mines Nantes, France \\ ${ }^{2}$ CNRS, University of Montpellier, France
}

\begin{abstract}
State-of-the-art constraint solvers uniformly maintain the same level of local consistency (usually arc consistency) on all the instances. We propose two approaches to adjust the level of consistency depending on the instance and on which part of the instance we propagate. The first approach, parameterized local consistency, uses as parameter the stability of values, which is a feature computed by arc consistency algorithms during their execution. Parameterized local consistencies choose to enforce arc consistency or a higher level of local consistency to a value depending on whether the stability of the value is above or below a given threshold. In the adaptive version, the parameter is dynamically adapted during search, and so is the level of local consistency. In the second approach, we focus on partition-one-AC, a singleton-based consistency. We propose adaptive variants of partition-one-AC that do not necessarily run until having proved the fixpoint. The pruning can be weaker than the full version, but the computational effort can be significantly reduced. Our experiments show that adaptive parameterized maxRPC and adaptive partition-one$\mathrm{AC}$ can obtain significant speed-ups over arc consistency and over the full versions of maxRPC and partition-one-AC.
\end{abstract}

\section{Introduction}

Enforcing local consistency by applying constraint propagation during search is one of the strengths of constraint programming $(\mathrm{CP})$. It allows the constraint solver to remove locally inconsistent values. This leads to a reduction of the search space. Arc consistency is the oldest and most well-known way of propagating constraints [Bes06]. It has the nice feature that it does not modify the structure of the constraint network. It just prunes infeasible values. Arc consistency is the standard level of consistency maintained in constraint solvers.

* The results contained in this chapter have been presented in [BBCB13] and [BBBT14]. This work has been funded by the EU project ICON (FP7-284715). 
Several other local consistencies pruning only values and stronger than arc consistency have been proposed, such as max restricted path consistency or singleton arc consistency [DB97]. These local consistencies are seldom used in practice because of the high computational cost of maintaining them during search. However, on some instances of problems, maintaining arc consistency is not a good choice because of the high number of ineffective revisions of constraints that penalize the CPU time. For instance, Stergiou observed that when solving the scen11, an instance from the radio link frequency assignment problem (RLFAP) class, with an algorithm maintaining arc consistency, only 27 out of the 4103 constraints of the problem were identified as causing a domain wipe-out and 1921 constraints did not prune any value [Ste09].

Choosing the right level of local consistency for solving a problem requires finding a good trade-off between the ability of this local consistency to remove inconsistent values, and the cost of the algorithm that enforces it. The works of [Ste08] and [PS12] suggest to take advantage of the power of strong propagation algorithms to reduce the search space while avoiding the high cost of maintaining them in the whole network. These methods result in a heuristic approach based on the monitoring of propagation events to dynamically adapt the level of local consistency (arc consistency or max restricted path consistency) to individual constraints. This prunes more values than arc consistency and less than max restricted path consistency. The level of propagation obtained is not characterized by a local consistency property. Depending on the order of propagation, we can converge on different closures. In other work, a high level of consistency is applied in a non exhaustive way, because it is very expensive when applied exhaustively everywhere in the network during the whole search. In [SS09], a preprocessing phase learns which level of consistency to apply on which parts of the instance. When dealing with global constraints, some authors propose to weaken arc consistency instead of strengthening it. In [KVH06], Katriel et al. proposed a randomized filtering scheme for AllDifferent and Global Cardinality Constraint. In [Sel03], Sellmann introduced the concept of approximated consistency for optimization constraints and provided filtering algorithms for Knapsack Constraints based on bounds with guaranteed accuracy.

In this chapter, we propose two approaches for adapting automatically the level of consistency during search. Our first approach is based on the notion of stability of values. This is an original notion independent of the characteristics of the instance to be solved, but based on the state of the arc consistency algorithm during its propagation. Based on this notion, we propose parameterized consistencies, an original approach to adjust the level of consistency inside a given instance. The intuition is that if a value is hard to prove arc consistent (i.e., the value is not stable for arc consistency), this value will perhaps be pruned by a stronger local consistency. The parameter $p$ specifies the threshold of stability of a value $v$ below which we will enforce a stronger consistency to $v$. A parameterized consistency $p$-LC is thus an intermediate level of consistency between arc consistency and another consistency LC, stronger than arc consistency. The strength of $p$-LC depends on the parameter $p$. This approach allows us to find a trade-off between the pruning power of local consistency and 
the computational cost of the algorithm that achieves it. We apply $p$-LC to the case where LC is max restricted path consistency. We describe the algorithm $p$ maxRPC3 (based on maxRPC3 [BPSW11]) that achieves $p$-max restricted path consistency. Then, we propose $a p$ - $\mathrm{LC}$, an adaptive variant of $p$-LC that uses the number of failures in which variables or constraints are involved to assess the difficulty of the different parts of the problem during search. ap-LC dynamically and locally adapts the level $p$ of local consistency to apply depending on this difficulty.

Our second approach is inspired by singleton-based consistencies. They have been shown extremely efficient to solve some classes of hard problems [BCDL11]. Singleton-based consistencies apply the singleton test principle, which consists of assigning a value to a variable and trying to refute it by enforcing a given level of consistency. If a contradiction occurs during this singleton test, the value is removed from its domain. The first example of such a local consistency is Singleton Arc Consistency (SAC), introduced in [DB97]. In SAC, the singleton test enforces arc consistency. By definition, SAC can only prune values in the variable domain on which it currently performs singleton tests. In [BA01], Partition-One-AC (which we call POAC) has been proposed. POAC is an extension of SAC that can prune values everywhere in the network as soon as a variable has been completely singleton tested. As a consequence, the fixpoint in terms of filtering is often quickly reached in practice. This observation has already been made on numerical constraint problems. In [TC07, NT13], a consistency called Constructive Interval Disjunction (CID), close to POAC in its principle, gave good results by simply calling the main procedure once on each variable or by adapting during search the number of times it is called. Based on these observations, we propose an adaptive version of POAC, called APOAC, where the number of times variables are processed for singleton tests on their values is dynamically and automatically adapted during search. A sequence of singleton tests on all values of one variable is called a varPOAC call. The number $k$ of times varPOAC is called will depend on how effective POAC is or not in pruning values. This number $k$ of varPOAC calls will be learned during a sequence of nodes of the search tree (learning nodes) by measuring stagnation in the amount of pruned values. This amount $k$ of varPOAC calls will be applied at each node during a sequence of nodes (called exploitation nodes) before we enter a new learning phase to adapt $k$ again. Observe that if the number of varPOAC calls learned is 0 , then adaptive POAC will mimic AC.

The aim of both of the proposed adaptive approaches (i.e., ap-LC and APOAC) is to adapt the level of consistency automatically and dynamically during search. $a p$-LC uses failure information to learn what are the most difficult parts of the problem and it increases locally and dynamically the parameter $p$ on those difficult parts. APOAC measures a stagnation in number of inconsistent values removed for $k$ calls of varPOAC. APOAC then uses this information to stop enforcing POAC. APOAC avoids the cost of the last calls to varPOAC that delete very few values or no value at all. We thus see that both $a p-\mathrm{LC}$ and APOAC learn some information during search to adapt the level of consistency. This allows them to benefit from the pruning power of a high level of consistency 
while avoiding the prohibitive time cost of fully maintaining this high level.

The rest of the paper is organized as follows. Section 2 contains the necessary formal background. Section 3 describes the parameterized consistency approach and gives an algorithm for parameterized maxRPC. In Section 4, the adaptive variant of parameterized consistency is defined. Sections 5 and 6 are devoted to our study of singleton-based consistencies. In Section 5, we propose an efficient POAC algorithm that will be used as a basis for the adaptive versions of POAC. Section 6 presents different ways to learn the number of variables on which to perform singleton tests. All these sections contain experimental results that validate the different contributions. Section 7 concludes this work.

\section{Background}

A constraint network is defined as a set of $n$ variables $X=\left\{x_{1}, \ldots, x_{n}\right\}$, a set of ordered domains $D=\left\{D\left(x_{1}\right), \ldots, D\left(x_{n}\right)\right\}$, and a set of $e$ constraints $C=\left\{c_{1}, \ldots, c_{e}\right\}$. Each constraint $c_{k}$ is defined by a pair $\left(\operatorname{var}\left(c_{k}\right), \operatorname{sol}\left(c_{k}\right)\right)$, where $\operatorname{var}\left(c_{k}\right)$ is an ordered subset of $X$, and $\operatorname{sol}\left(c_{k}\right)$ is a set of combinations of values (tuples) satisfying $c_{k}$. In the following, we restrict ourselves to binary constraints, because the local consistency (maxRPC) we use here to instantiate our approach is defined on the binary case only. However, the notions we introduce can be extended to non-binary constraints, by using maxRPWC for instance [BSW08]. A binary constraint $c$ between $x_{i}$ and $x_{j}$ will be denoted by $c_{i j}$, and $\Gamma\left(x_{i}\right)$ will denote the set of variables $x_{j}$ involved in a constraint with $x_{i}$.

A value $v_{j} \in D\left(x_{j}\right)$ is called an arc consistent support (AC support) for $v_{i} \in D\left(x_{i}\right)$ on $c_{i j}$ if $\left(v_{i}, v_{j}\right) \in \operatorname{sol}\left(c_{i j}\right)$. A value $v_{i} \in D\left(x_{i}\right)$ is arc consistent (AC) if and only if for all $x_{j} \in \Gamma\left(x_{i}\right) v_{i}$ has an AC support $v_{j} \in D\left(x_{j}\right)$ on $c_{i j}$. A domain $D\left(x_{i}\right)$ is arc consistent if it is non empty and all values in $D\left(x_{i}\right)$ are arc consistent. A network is arc consistent if all domains in $D$ are arc consistent. If enforcing arc consistency on a network $N$ leads to a domain wipe out, we say that $N$ is arc inconsistent.

A tuple $\left(v_{i}, v_{j}\right) \in D\left(x_{i}\right) \times D\left(x_{j}\right)$ is path consistent (PC) if and only if for any third variable $x_{k}$ there exists a value $v_{k} \in D\left(x_{k}\right)$ such that $v_{k}$ is an AC support for both $v_{i}$ and $v_{j}$. In such a case, $v_{k}$ is called witness for the path consistency of $\left(v_{i}, v_{j}\right)$.

A value $v_{j} \in D\left(x_{j}\right)$ is a max restricted path consistent (maxRPC) support for $v_{i} \in D\left(x_{i}\right)$ on $c_{i j}$ if and only if it is an AC support and the tuple $\left(v_{i}, v_{j}\right)$ is path consistent. A value $v_{i} \in D\left(x_{i}\right)$ is max restricted path consistent on a constraint $c_{i j}$ if and only if there exist $v_{j} \in D\left(x_{j}\right)$ maxRPC support for $v_{i}$ on $c_{i j}$. A value $v_{i} \in D\left(x_{i}\right)$ is max restricted path consistent ifand only if for all $x_{j} \in \Gamma\left(x_{i}\right) v_{i}$ has a maxRPC support $v_{j} \in D\left(x_{j}\right)$ on $c_{i j}$. A variable $x_{i}$ is maxRPC if its domain $D\left(x_{i}\right)$ is non empty and all values in $D\left(x_{i}\right)$ are maxRPC. A network is maxRPC if all domains in $D$ are maxRPC.

A value $v_{i} \in D\left(x_{i}\right)$ is singleton arc consistent (SAC) if and only if the network $\left.N\right|_{x_{i}=v_{i}}$ where $D\left(x_{i}\right)$ is reduced to the singleton $\left\{v_{i}\right\}$ is not arc incon- 
sistent. A variable $x_{i}$ is SAC if $D\left(x_{i}\right) \neq \emptyset$ and all values in $D\left(x_{i}\right)$ are SAC. A network is SAC if all its variables are SAC.

A variable $x_{i}$ is partition-one-AC (POAC) if and only if $D\left(x_{i}\right) \neq \emptyset$, all values in $D\left(x_{i}\right)$ are SAC, and $\forall j \in 1 . . n, j \neq i, \forall v_{j} \in D\left(x_{j}\right), \exists v_{i} \in D\left(x_{i}\right)$ such that $v_{j} \in A C\left(\left.N\right|_{x_{i}=v_{i}}\right)$. A constraint network $N=(X, D, C)$ is POAC if and only if all its variables are POAC. Observe that POAC, as opposed to SAC, is able to prune values from all variable domains when being enforced on a given variable.

Following [DB97], we say that a local consistency $L C_{1}$ is stronger than a local consistency $L C_{2}\left(L C_{2} \preceq L C_{1}\right)$ if $L C_{2}$ holds on any constraint network on which $L C_{1}$ holds. It has been shown in [BA01] that POAC is strictly stronger than SAC. Hence, SAC holds on any constraint network on which POAC holds and there exist constraint networks on which SAC holds but not POAC.

The problem of deciding whether a constraint network has solutions is called the constraint satisfaction problem (CSP), and it is NP-complete. Solving a CSP is mainly done by backtrack search that maintains some level of consistency between each branching step.

\section{Parameterized Consistency}

In this section, we present an original approach to parameterize a level of consistency LC stronger than arc consistency so that it degenerates to arc consistency when the parameter equals 0 , to $\mathrm{LC}$ when the parameters equals 1 , and to levels in between when the parameter is between 0 and 1 . The idea behind this is to be able to adjust the level of consistency to the instance to be solved, hoping that such an adapted level of consistency will prune significantly more values than arc consistency while being less time consuming than LC.

Parameterized consistency is based on the concept of stability of values. We first need to define the 'distance to end' of a value in a domain. This captures how far a value is from the last in its domain. In the following, $\operatorname{rank}(v, S)$ is the position of value $v$ in the ordered set of values $S$.

Definition 1 (Distance to end of a value) The distance to end of a value $v_{i} \in D\left(x_{i}\right)$ is the ratio

$$
\Delta\left(x_{i}, v_{i}\right)=\left(\left|D_{o}\left(x_{i}\right)\right|-\operatorname{rank}\left(v_{i}, D_{o}\left(x_{i}\right)\right)\right) /\left|D_{o}\left(x_{i}\right)\right|,
$$

where $D_{o}\left(x_{i}\right)$ is the initial domain of $x_{i}$.

We see that the first value in $D_{o}\left(x_{i}\right)$ has distance $\left(\left|D_{o}\left(x_{i}\right)\right|-1\right) /\left|D_{o}\left(x_{i}\right)\right|$ and the last one has distance 0 . Thus, $\forall v_{i} \in D\left(x_{i}\right), 0 \leq \Delta\left(x_{i}, v_{i}\right)<1$.

We can now give the definition of what we call the parameterized stability of a value for arc consistency. The idea is to define stability for values based on the distance to the end of their AC supports. For instance, consider the constraint $x_{1} \leq x_{2}$ with the domains $D\left(x_{1}\right)=D\left(x_{2}\right)=\{1,2,3,4\}$ (see Figure 1). $\Delta\left(x_{2}, 1\right)=(4-1) / 4=0.75, \Delta\left(x_{2}, 2\right)=0.5, \Delta\left(x_{2}, 3\right)=0.25$ and $\Delta\left(x_{2}, 4\right)=0$. 


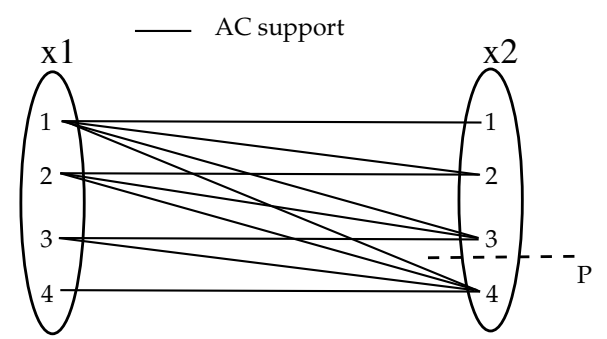

Figure 1: Stability of supports on the example of the constraint $x_{1} \leq x_{2}$ with the domains $D\left(x_{1}\right)=D\left(x_{2}\right)=\{1,2,3,4\}$. $\left(x_{1}, 4\right)$ is not p-stable for AC.

If $p=0.2$, the value $\left(x_{1}, 4\right)$ is not $p$-stable for $\mathrm{AC}$, because the first and only AC support of $\left(x_{1}, 4\right)$ in the ordering used to look for supports, that is $\left(x_{2}, 4\right)$, has a distance to end smaller than the threshold $p$. Proving that the pair $(4,4)$ is inconsistent (by a stronger consistency) could lead to the pruning of $\left(x_{1}, 4\right)$. In other words, applying a stronger consistency on $\left(x_{1}, 4\right)$ has a higher chance to lead to its removal than applying it to for instance $\left(x_{1}, 1\right)$, which had no difficulty to find its first AC support (distance to end of $\left(x_{2}, 1\right)$ is 0.75 ).

At this point, we want to emphasize that the ordering of values used to look for supports in the domains is not related to the order in which values are selected by the branching heuristic used by the backtrack search procedure. That is, we can use a given order of values for looking for supports and another one for exploring the search tree.

Definition 2 ( $p$-stability for AC) A value $v_{i} \in D\left(x_{i}\right)$ is p-stable for $A C$ on $c_{i j}$ iff $v_{i}$ has an $A C$ support $v_{j} \in D\left(x_{j}\right)$ on $c_{i j}$ such that $\Delta\left(x_{j}, v_{j}\right) \geq p$. A value $v_{i} \in D\left(x_{i}\right)$ is p-stable for $A C$ iff $\forall x_{j} \in \Gamma\left(x_{i}\right)$, $v_{i}$ is p-stable for $A C$ on $c_{i j}$.

We are now ready to give the first definition of parameterized local consistency. This first definition can be applied to any local consistency LC for which the consistency of a value on a constraint is well defined. This is the case for instance for all triangle-based consistencies [DB01, Bes06].

Definition 3 (Constraint-based $p$-LC) Let LC be a local consistency stronger than $A C$ for which the $L C$ consistency of a value on a constraint is defined. A value $v_{i} \in D\left(x_{i}\right)$ is constraint-based $p$-LC on $c_{i j}$ iff it is $p$-stable for $A C$ on $c_{i j}$, or it is $L C$ on $c_{i j}$. A value $v_{i} \in D\left(x_{i}\right)$ is constraint-based $p-L C$ iff $\forall c_{i j}, v_{i}$ is constraint-based $p-L C$ on $c_{i j}$. A constraint network is constraint-based $p$-LC iff all values in all domains in $D$ are constraint-based $p-L C$.

Theorem 1 Let $L C$ be a local consistency stronger than $A C$ for which the $L C$ consistency of a value on a constraint is defined. Let $p_{1}$ and $p_{2}$ be two parameters in [0..1]. If $p_{1}<p_{2}$, then $A C \preceq$ constraint-based $p_{1}-L C \preceq$ constraint-based $p_{2}$ $L C \preceq L C$. 
Proof. Suppose that there exist two parameters $p_{1}, p_{2}$ such that $0 \leq p_{1}<p_{2} \leq$ 1 , and suppose that there exists a $p_{2}$-LC constraint network $N$ that contains a $p_{2}$-LC value $\left(x_{i}, v_{i}\right)$ that is $p_{1}-\mathrm{LC}$ inconsistent. Let $c_{i j}$ be the constraint on which $\left(x_{i}, v_{i}\right)$ is $p_{1}$-LC inconsistent. Then, $\nexists v_{j} \in D\left(x_{j}\right)$ that is an AC support for $\left(x_{i}, v_{i}\right)$ on $c_{i j}$ such that $\Delta\left(x_{j}, v_{j}\right) \geq p_{1}$. Thus, $v_{i}$ is not $p_{2}$-stable for AC on $c_{i j}$. In addition, $v_{i}$ is not $\mathrm{LC}$ on $c_{i j}$. Therefore, $v_{i}$ is not $p_{2}-\mathrm{LC}$, and $N$ is not $p_{2}$-LC.

Definition 3 can be modified to a more coarse-grained version that is not dependent on the consistency of values on a constraint. This will have the advantage to apply to any type of strong local consistency, even those, like singleton arc consistency, for which the consistency of a value on a constraint is not defined.

Definition 4 (Value-based $p$-LC) Let $L C$ be a local consistency stronger than $A C$. A value $v_{i} \in D\left(x_{i}\right)$ is value-based $p-L C$ if and only if it is p-stable for $A C$ or it is LC. A constraint network is value-based $p$ - LC if and only if all values in all domains in $D$ are value-based $p-L C$.

Theorem 2 Let $L C$ be a local consistency stronger than AC. Let $p_{1}$ and $p_{2}$ be two parameters in [0..1]. If $p_{1}<p_{2}$ then $A C \preceq$ value-based $p_{1}-L C \preceq$ value-based $p_{2}-L C \preceq L C$.

Proof. Suppose that there exist two parameters $p_{1}, p_{2}$ such that $0 \leq p_{1}<p_{2} \leq$ 1 , and suppose that there exists a $p_{2}-\mathrm{LC}$ constraint network $N$ that contains a $p_{2}$-LC value $\left(x_{i}, v_{i}\right)$ that is $p_{1}$-LC-inconsistent. $v_{i}$ is $p_{1}$-LC-inconsistent means that:

1. $v_{i}$ is not $p_{1}$-stable for AC: $\exists c_{i j}$ on which $v_{i}$ is not $p_{1}$-stable for AC. Then $\nexists v_{j} \in D\left(x_{j}\right)$ that is an $\mathrm{AC}$ support for $\left(x_{i}, v_{i}\right)$ on $c_{i j}$ such that $\Delta\left(x_{j}, v_{j}\right) \geq$ $p_{1}$. Therefore, $v_{i}$ is not $p_{2}$-stable for AC on $c_{i j}$, then $v_{i}$ is not $p_{2}$-stable for AC.

2. $v_{i}$ is LC inconsistent.

(1) and (2) imply that $v_{i}$ is not $p_{2}$-LC and $N$ is not $p_{2}$-LC.

For both types of definitions of $p$-LC, we have the following property on the extreme cases $(p=0, p=1)$.

Corollary 1 Let $L C_{1}$ and $L C_{2}$ be two local consistencies stronger than $A C$. We have: value-based $0-L C_{2}=A C$ and value-based $1-L C_{2}=L C$. If the $L C_{1}$ consistency of a value on a constraint is defined, we also have: constraint-based $0-L C_{1}=A C$ and constraint-based 1-LC $=L C$.

\subsection{Parameterized maxRPC: $p$-maxRPC}

To illustrate the benefit of our approach, we apply parameterized consistency to maxRPC to obtain the $p$-maxRPC level of consistency that achieves a consistency level between AC and maxRPC. 


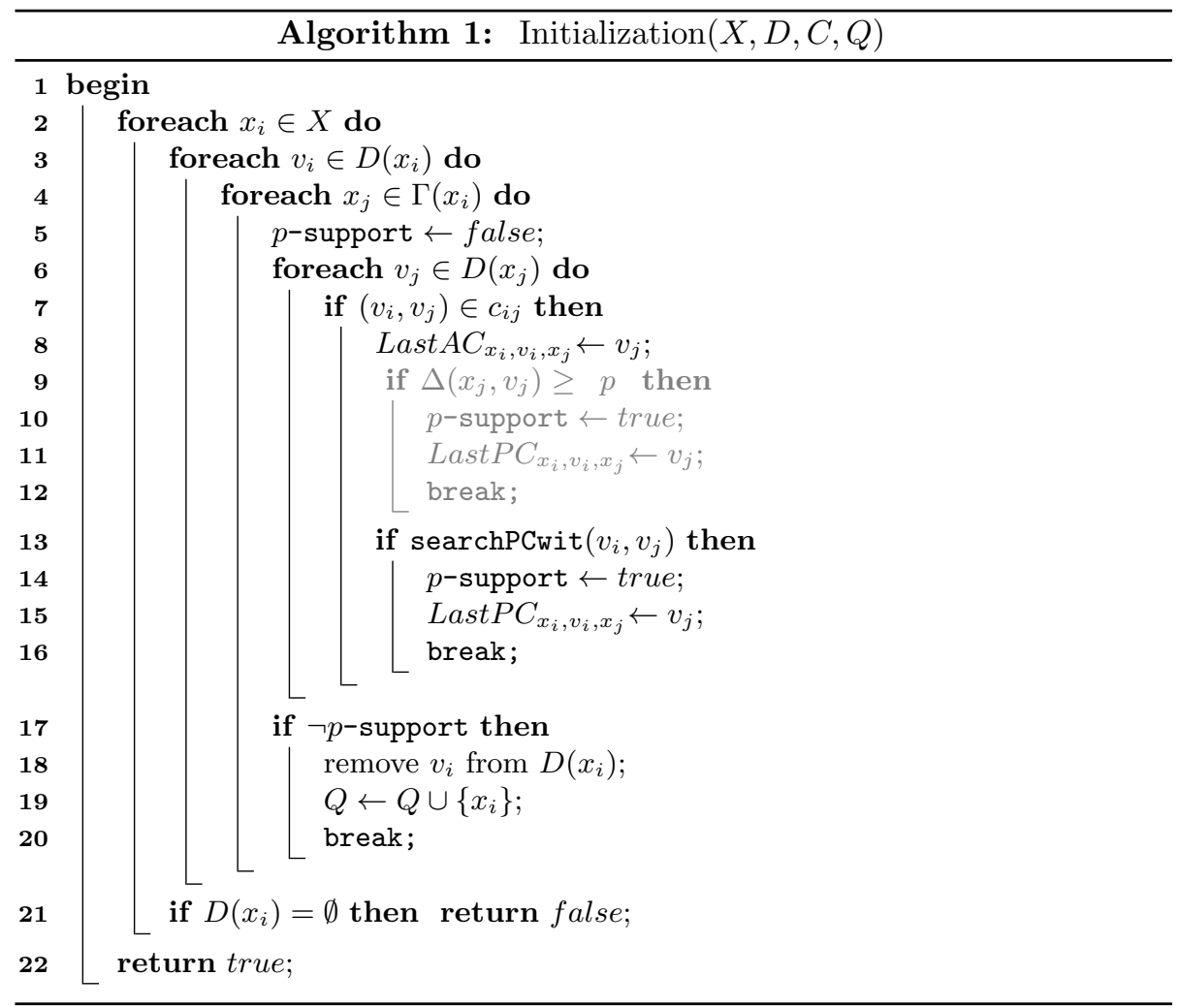

Definition 5 ( $p$-maxRPC) $A$ value is $p$-maxRPC if and only if it is constraint-based $p$-maxRPC. A network is $p$-maxRPC if and only if it is constraint-based $p$-maxRPC.

From Theorem 1 and Corollary 1 we derive the following corollary.

Corollary 2 For any two parameters $p_{1}, p_{2}, 0 \leq p_{1}<p_{2} \leq 1, A C \preceq p_{1}$ $\max R P C \preceq p_{2}-\max R P C \preceq \max R P C .0-\max R P C=A C$ and $1-\max R P C=$ $\max R P C$.

We propose an algorithm for $p$-maxRPC, based on maxRPC3, the best existing maxRPC algoritm. We do not describe maxRPC3 in full detail, as it can be found in [BPSW11]. We only describe procedures where changes to maxRPC3 are necessary to design $p$-maxRPC3, a coarse grained algorithm that performs $p$-maxRPC. We use light grey to emphasize the modified parts of the original maxRPC3 algorithm.

maxRPC3 uses a propagation list $Q$ where it inserts the variables whose domains have changed. It also uses two other data structures: LastAC and LastPC. For each value $\left(x_{i}, v_{i}\right)$, LastAC $x_{x_{i}, v_{i}, x_{j}}$ stores the smallest AC support for $\left(x_{i}, v_{i}\right)$ on $c_{i j}$ and LastPC $x_{i}, v_{i}, x_{j}$ stores the smallest PC support for $\left(x_{i}, v_{i}\right)$ 


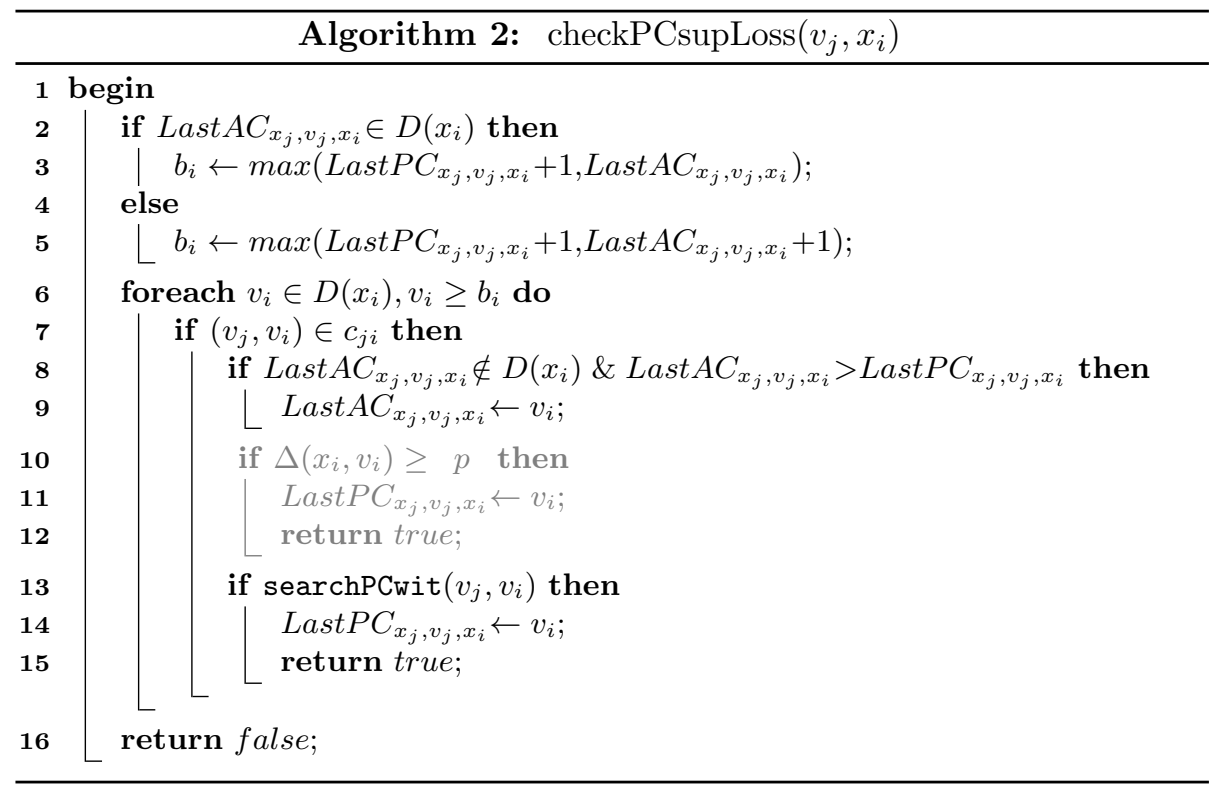

on $c_{i j}$ (i.e., the smallest AC support $\left(x_{j}, v_{j}\right)$ for $\left(x_{i}, v_{i}\right)$ on $c_{i j}$ such that $\left(v_{i}, v_{j}\right)$ is PC). This algorithm comprises two phases: initialization and propagation.

In the initialization phase (algorithm 1) maxRPC3 checks if each value $\left(x_{i}, v_{i}\right)$ has a maxRPC-support $\left(x_{j}, v_{j}\right)$ on each constraint $c_{i j}$. If not, it removes $v_{i}$ from $D\left(x_{i}\right)$ and inserts $x_{i}$ in $Q$. To check if a value $\left(x_{i}, v_{i}\right)$ has a maxRPC-support on a constraint $c_{i j}$, maxRPC3 looks first for an AC-support $\left(x_{j}, v_{j}\right)$ for $\left(x_{i}, v_{i}\right)$ on $c_{i j}$, then it checks if $\left(v_{i}, v_{j}\right)$ is PC. In this last step, changes were necessary to obtain $p$-maxRPC3 (lines 9-12). We check if $\left(v_{i}, v_{j}\right)$ is $\mathrm{PC}$ (line 13) only if $\Delta\left(x_{j}, v_{j}\right)$ is smaller than the parameter $p$ (line 9 ).

The propagation phase of maxRPC3 involves propagating the effect of deletions. While $Q$ is non empty, maxRPC3 extracts a variable $x_{i}$ from $Q$ and checks for each value $\left(x_{j}, v_{j}\right)$ of each neighboring variable $x_{j} \in \Gamma\left(x_{i}\right)$ if it is not maxRPC because of deletions of values in $D\left(x_{i}\right)$. A value $\left(x_{j}, v_{j}\right)$ becomes maxRPC inconsistent in two cases: if its unique PC-support $\left(x_{i}, v_{i}\right)$ on $c_{i j}$ has been deleted, or if we deleted the unique witness $\left(x_{i}, v_{i}\right)$ for a pair $\left(v_{j}, v_{k}\right)$ such that $\left(x_{k}, v_{k}\right)$ is the unique PC-support for $\left(x_{j}, v_{j}\right)$ on $c_{j k}$. So, to propagate deletions, maxRPC3 checks if the last maxRPC support (last known support) of $\left(x_{j}, v_{j}\right)$ on $c_{i j}$ still belongs to the domain of $x_{i}$, otherwise it looks for the next support (algorithm 2). If such a support does not exist, it removes the value $v_{j}$ and adds the variable $x_{j}$ to $Q$. Then if $\left(x_{j}, v_{j}\right)$ has not been removed in the previous step, maxRPC3 checks (algorithm 3) whether there is still a witness for each pair $\left(v_{j}, v_{k}\right)$ such that $\left(x_{k}, v_{k}\right)$ is the PC support for $\left(x_{j}, v_{j}\right)$ on $c_{j k}$. If not, it looks for the next maxRPC support for $\left(x_{j}, v_{j}\right)$ on $c_{j k}$. If such a support does not exist, it removes $v_{j}$ from $D\left(x_{j}\right)$ and adds the variable $x_{j}$ to $Q$. 


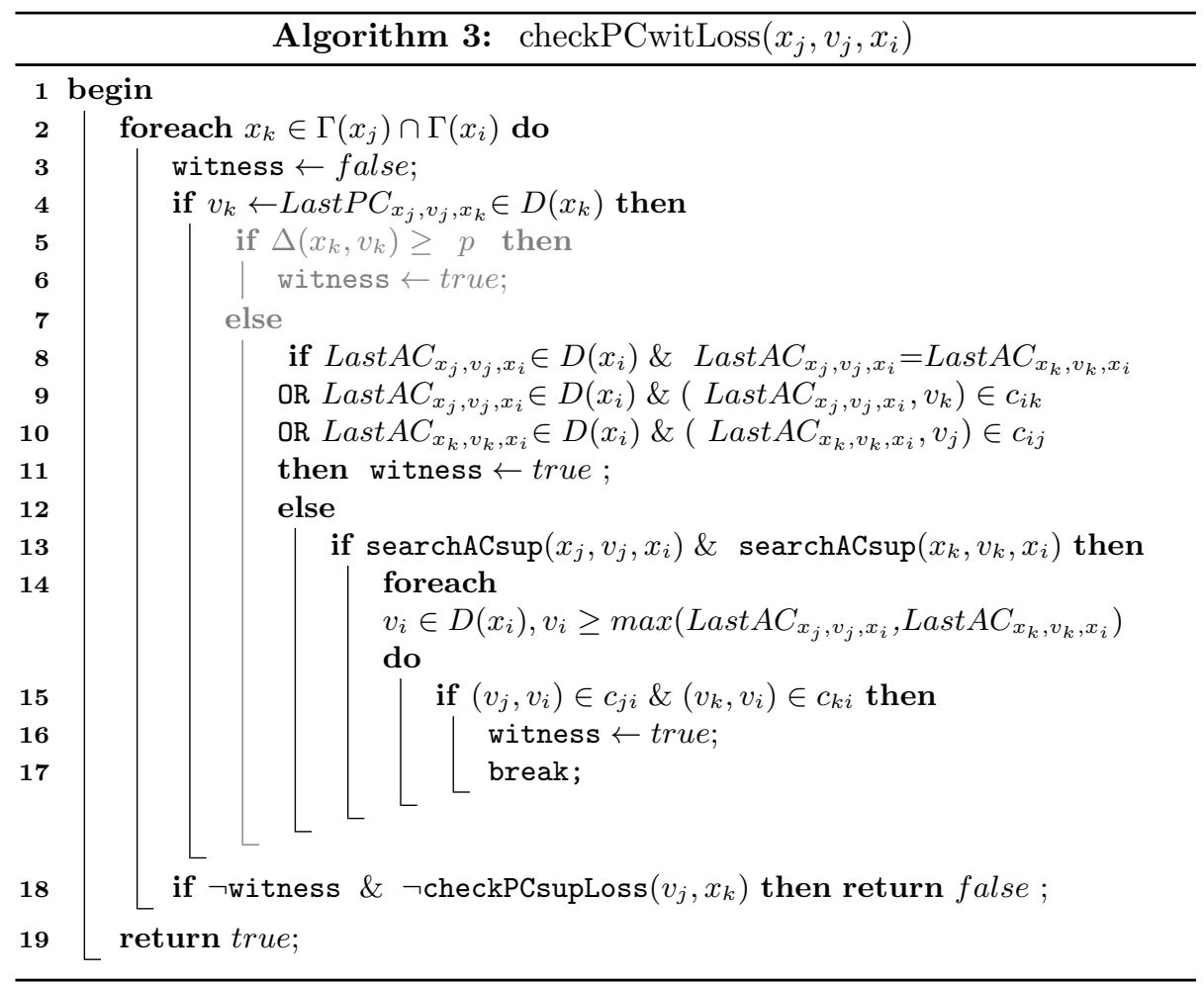

In the propagation phase, we also modified maxRPC 3 to check if the values are still $p$-maxRPC instead of checking if they are maxRPC. In $p$-maxRPC3, the last $p$-maxRPC support for $\left(x_{j}, v_{j}\right)$ on $c_{i j}$ is the last AC support if $\left(x_{j}, v_{j}\right)$ is $p$-stable for $\mathrm{AC}$ on $c_{i j}$. If not, it is the last PC support. Thus, $p$-maxRPC3 checks if the last $p$-maxRPC support (last known support) of $\left(x_{j}, v_{j}\right)$ on $c_{i j}$ still belongs to the domain of $x_{i}$. If not, it looks (algorithm 2) for the next AC support $\left(x_{i}, v_{i}\right)$ on $c_{i j}$, and checks if $\left(v_{i}, v_{j}\right)$ is PC (line 13) only when $\Delta\left(x_{i}, v_{i}\right)<p$ (line 10). If no $p$-maxRPC support exists, $p$-maxRPC3 removes the value and adds the variable $x_{j}$ to $Q$. If the value $\left(x_{j}, v_{j}\right)$ has not been removed in the previous phase, $p$-maxRPC3 checks (algorithm 3 ) whether there is still a witness for each pair $\left(v_{j}, v_{k}\right)$ such that $\left(x_{k}, v_{k}\right)$ is the $p$-maxRPC support for $v_{j}$ on $c_{j k}$ and $\Delta\left(x_{k}, v_{k}\right)<p$. If not, it looks for the next $p$-maxRPC support for $v_{j}$ on $c_{j k}$. If such a support does not exist, it removes $v_{j}$ from $D\left(x_{j}\right)$ and adds the variable $x_{j}$ to $Q$.

$p$-maxRPC3 uses the data structure LastPC to store the last $p$-maxRPC support (i.e., the latest $\mathrm{AC}$ support for the $p$-stable values and the latest $\mathrm{PC}$ support for the others). Algorithms 1 and 2 update the data structure LastPC of maxRPC3 to be LastAC for all the values that are $p$-stable for AC (line 11 of Algorithm 1 and line 11 of Algorithm 2) and avoid seeking witnesses for those values. Algorithm 3 avoids checking the loss of witnesses for the $p$-stable 
values by setting the flag witness to true (line 6). Correctness of $p$-maxRPC3 directly comes from maxRPC3: The removed values are necessarily $p$-maxRPCinconsistent and all the values that are $p$-maxRPC-inconsistent are removed.

\subsection{Experimental validation of $p$-maxRPC}

To validate the approach of parameterized local consistency, we conducted a first basic experiment. The purpose of this experiment is to see if there exist instances on which a given level of $p$-maxRPC, with a value $p$ that is uniform (i.e., identical for the entire constraint network) and static (i.e., constant through the entire search process), is more efficient than $\mathrm{AC}$ or maxRPC, or both.

We have implemented the algorithms that achieve $p$-maxRPC as described in the previous section in our own binary constraint solver, in addition to maxRPC (maxRPC3 version [BPSW11]) and AC (AC2001 version [BRYZ05]). All the algorithms are implemented in our JAVA CSP solver.We tested these algorithms on several classes of CSP instances from the International Constraint Solver Competition $09^{1}$. We have only selected instances involving binary constraints. To isolate the effect of propagation, we used the lexicographic ordering for variables and values. We set the CPU timeout to one hour. Our experiments were conducted on a 12-core Genuine Intel machine with 16Gb of RAM running at $2.92 \mathrm{GHz}$.

On each instance of our experiment, we ran AC, max-RPC, and $p$-maxRPC for all values of $p$ in $\{0.1,0.2, \ldots, 0.9\}$. Performance has been measured in terms of CPU time in seconds, the number of visited nodes (NODE) and the number of constraint checks (CCK). Results are presented as "CPU time $(p)$ ", where $p$ is the parameter for which $p$-maxRPC gives the best result.

Table 1 reports the performance of $\mathrm{AC}$, maxRPC, and $p$-maxRPC for the value of $p$ producing the best CPU time, on instances from Radio Link Frequency Assignment Problems (RLFAPs), geom problems, and queens knights problems. The CPU time of the best algorithm is bold-faced. On RLFAP and geom, we observe the existence of a parameter $p$ for which $p$-maxRPC is faster than both $\mathrm{AC}$ and maxRPC for most instances of these two classes of problems. On the queens-knight problem, however, $\mathrm{AC}$ is always the best algorithm. In Figures 2 and 3 , we try to understand more closely what makes $p$-maxRPC better or worse than AC and maxRPC. Figures 2 and 3 plot the performance (CPU, NODE and CCK) of $p$-maxRPC for all values of $p$ from 0 to 1 by steps of 0.1 against performance of $\mathrm{AC}$ and maxRPC. Figure 2 shows an instance where $p$-maxRPC solves the problem faster than $\mathrm{AC}$ and maxRPC for values of $p$ in the range [0.3..0.8]. We observe that $p$-maxRPC is faster than AC and maxRPC when it reduces the size of the search space as much as maxRPC (same number of nodes visited) with a number of CCK closer to the number of CCK produced by AC. Figure 3 shows an instance where the CPU time for $p$-maxRPC is never better than both $\mathrm{AC}$ and maxRPC, whatever the value of $p$. We see that $p$ maxRPC is two to three times faster than maxRPC. But $p$-maxRPC fails to

\footnotetext{
${ }^{1}$ http://cpai.ucc.ie/09/
} 
Table 1: Performance (CPU time, nodes and constraint checks) of AC, $p$ maxRPC, and maxRPC on various instances.

\begin{tabular}{|c|c|c|c|c|c|}
\hline & & $\mathbf{A C}$ & $p$-maxRPC & & $\max R P C$ \\
\hline \multirow[t]{3}{*}{ scen1-f8 } & $\mathrm{CPU}$ & $>3600$ & 1.39 & $(0.2)$ & 6.10 \\
\hline & \#nodes & - & 927 & & 917 \\
\hline & \#ccks & - & $1,397,440$ & & $26,932,990$ \\
\hline \multirow[t]{3}{*}{ scen2-f24 } & $\mathrm{CPU}$ & $>3600$ & 0.13 & $(0.3)$ & 0.65 \\
\hline & \#nodes & - & 201 & & 201 \\
\hline & \#ccks & - & 296,974 & & $3,462,070$ \\
\hline \multirow[t]{3}{*}{ scen3-f10 } & $\mathrm{CPU}$ & $>3600$ & 0.89 & $(0.5)$ & 2.80 \\
\hline & \#nodes & - & 469 & & 408 \\
\hline & \#ccks & - & 874,930 & & $13,311,797$ \\
\hline \multirow[t]{3}{*}{ geo50-20-d4-75-26 } & $\mathrm{CPU}$ & 111.48 & 17.80 & $(1.0)$ & $\overline{15.07}$ \\
\hline & \#nodes & 477,696 & 3,768 & & 3,768 \\
\hline & \#ccks & $96,192,822$ & $40,784,017$ & & $40,784,017$ \\
\hline \multirow[t]{3}{*}{ geo50-20-d4-75-43 } & $\mathrm{CPU}$ & $1,671.35$ & $1,264.36$ & $(0.5)$ & $1,530.02$ \\
\hline & \#nodes & $4,118,134$ & 555,259 & & 279,130 \\
\hline & \#ccks & $1,160,664,461$ & $1,801,402,535$ & & $3,898,964,831$ \\
\hline \multirow[t]{3}{*}{ geo50-20-d4-75-46 } & CPU & $1,732.22$ & 371.30 & $(0.6)$ & 517.35 \\
\hline & \#nodes & $3,682,394$ & 125,151 & & 64,138 \\
\hline & \#ccks & $1,516,856,615$ & $584,743,023$ & & $1,287,674,430$ \\
\hline \multirow{3}{*}{ geo50-20-d4-75-84 } & $\mathrm{CPU}$ & 404.63 & 0.44 & $(0.6)$ & 0.56 \\
\hline & \#nodes & $2,581,794$ & 513 & & 333 \\
\hline & \#ccks & $293,092,144$ & 800,657 & & $1,606,047$ \\
\hline \multirow[t]{3}{*}{ queensKnights10-5-add } & $\mathrm{CPU}$ & 27.14 & 30.79 & $(0.2)$ & 98.44 \\
\hline & \#nodes & 82,208 & 81,033 & & 78,498 \\
\hline & $\#$ ccks & $131,098,933$ & $148,919,686$ & & $954,982,880$ \\
\hline \multirow[t]{3}{*}{ queensKnights10-5-mul } & $\mathrm{CPU}$ & 43.89 & 83.27 & $(0.1)$ & 300.74 \\
\hline & \#nodes & 74,968 & 74,414 & & 70,474 \\
\hline & \#ccks & $104,376,698$ & $140,309,576$ & & $1,128,564,278$ \\
\hline
\end{tabular}

improve AC because the number of constraint checks performed by $p$-maxRPC is much higher than the number of constraint checks performed by AC, whereas the number of nodes visited by $p$-maxRPC is not significantly reduced compared to the number of nodes visited by AC. From these observations, it thus seems that $p$-maxRPC outperforms $\mathrm{AC}$ and maxRPC when it finds a compromise between the number of nodes visited (the power of maxRPC) and the number of CCK needed to maintain (the light cost of AC).

In Figures 2 and 3 we can see that the CPU time for 1-maxRPC (respectively 0-maxRPC) is greater than the CPU time for maxRPC (respectively AC), although the two consistencies are equivalent. The reason is that $p$-maxRPC performs tests on the distances. For $p=0$, we also explain this difference by the fact that $p$-maxRPC maintains data structures that $\mathrm{AC}$ does not use.

\section{Adaptative Parameterized Consistency: ap- $\operatorname{maxRPC}$}

In the previous section, we have defined $p$-maxRPC, a version of parameterized consistency where the strong local consistency is maxRPC. We have performed some initial experiments where $p$ has the same value during the whole search and 


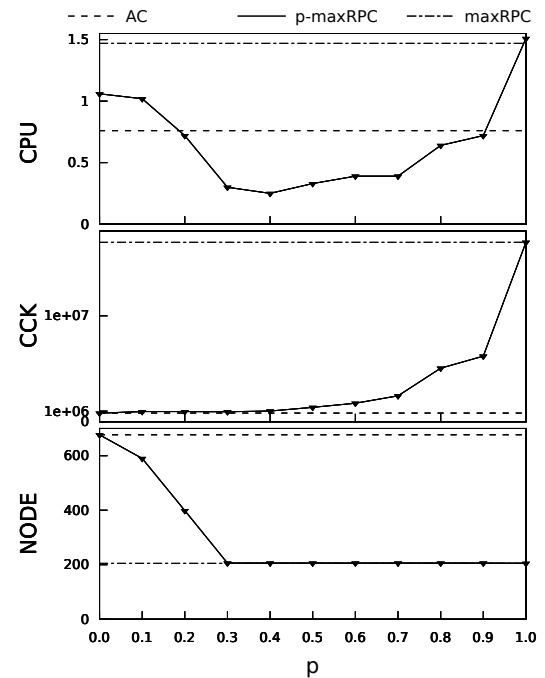

Figure 2: Instance where $p$-maxRPC outperforms both AC and maxRPC.

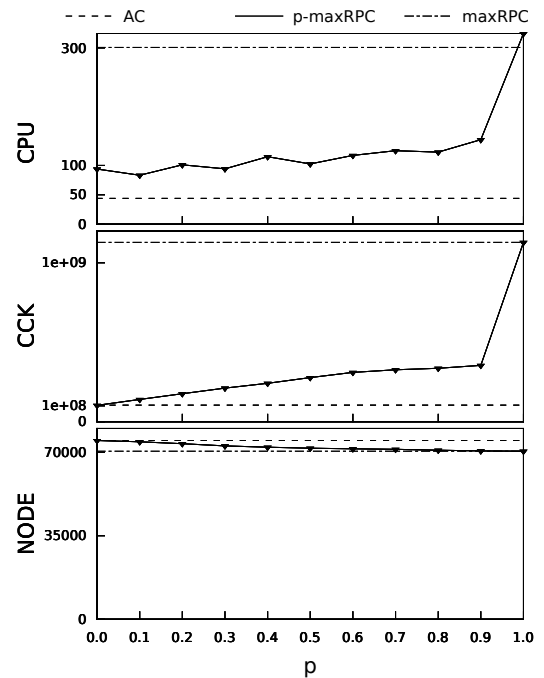

Figure 3: Instance where AC outperforms $p$-maxRPC.

everywhere in the constraint network. However, the algorithm we proposed to enforce $p$-maxRPC does not specify how $p$ is chosen. In this section, we propose two possible ways to dynamically and locally adapt the parameter $p$ in order to solve the problem faster than both AC and maxRPC. Instead of using a single value for $p$ during the whole search and for the whole constraint network, we propose to use several local parameters and to adapt the level of local consistency by dynamically adjusting the value of the different local parameters during search. The idea is to concentrate the effort of propagation by increasing the level of consistency in the most difficult parts of the given instance. We can determine these difficult parts using heuristics based on conflicts in the same vein as the weight of a constraint or the weighted degree of a variable in [BHLS04].

\subsection{Constraint-based $a p$-maxRPC : $a p c-\operatorname{maxRPC}$}

The first technique we propose, called constraint-based ap-maxRPC, assigns a parameter $p\left(c_{k}\right)$ to each constraint $c_{k}$ in $C$. We define this parameter to be correlated to the weight of the constraint. The idea is to apply a higher level of consistency in parts of the problem where the constraints are the most active.

Definition 6 (The weight of a constraint [BHLS04]) The weight $w\left(c_{k}\right)$ of a constraint $c_{k} \in C$ is an integer that is incremented every time a domain wipe-out occurs while performing propagation on this constraint.

We define the adaptive parameter $p\left(c_{k}\right)$ local to constraint $c_{k}$ in such a way that it is greater when the weight $w\left(c_{k}\right)$ is higher w.r.t. other constraints. 


$$
\forall c_{k} \in C, p\left(c_{k}\right)=\frac{w\left(c_{k}\right)-\min _{c \in C}(w(c))}{\max _{c \in C}(w(c))-\min _{c \in C}(w(c))}
$$

Equation 1 is normalized so that we are guaranteed that $0 \leq p\left(c_{k}\right) \leq 1$ for all $c_{k} \in C$ and that there exists $c_{k_{1}}$ with $p\left(c_{k_{1}}\right)=0$ (the constraint with lowest weight) and $c_{k_{2}}$ with $p\left(c_{k_{2}}\right)=1$ (the constraint with highest weight).

We are now ready to define adaptive parameterized consistency based on constraints.

Definition 7 (constraint-based ap-maxRPC) $A$ value $v_{i} \in D\left(x_{i}\right)$ is constraint-based ap-maxRPC (or apc-maxRPC) on a constraint $c_{i j}$ if and only if it is constraint-based $p\left(c_{i j}\right)$-maxRPC. A value $v_{i} \in D\left(x_{i}\right)$ is apc-maxRPC iff $\forall c_{i j}, v_{i}$ is apc-maxRPC on $c_{i j}$. A constraint network is apc-maxRPC iff all values in all domains in $D$ are apc-maxRPC.

\subsection{Variable-based $a p$-maxRPC: $a p x-\operatorname{maxRPC}$}

The technique proposed in Section 4.1 can only be used on consistencies where the consistency of a value on a constraint is defined. We present a second technique which can be used on constraint-based or variable-based local consistencies indifferently. We instantiate our definitions to maxRPC but the extension to other consistencies is direct. We call this new technique variable-based apmaxRPC. We need to define the weighted degree of a variable as the aggregation of the weights of all constraints involving it.

Definition 8 (The weighted degree of a variable [BHLS04]) The weighted degree wdeg $\left(x_{i}\right)$ of a variable $x_{i}$ is the sum of the weights of the constraints involving $x_{i}$ and one other uninstantiated variable.

We associate each variable with an adaptive local parameter based on its weighted degree.

$$
\forall x_{i} \in X, p\left(x_{i}\right)=\frac{w \operatorname{deg}\left(x_{i}\right)-\min _{x \in X}(w \operatorname{deg}(x))}{\max _{x \in X}(w \operatorname{deg}(x))-\min _{x \in X}(w \operatorname{deg}(x))}
$$

As in Equation 1, we see that the local parameter is normalized so that we are guaranteed that $0 \leq p\left(x_{i}\right) \leq 1$ for all $x_{i} \in X$ and that there exists $x_{k_{1}}$ with $p\left(x_{k_{1}}\right)=0$ (the variable with lowest weighted degree) and $x_{k_{2}}$ with $p\left(x_{k_{2}}\right)=1$ (the variable with highest weighted degree).

Definition 9 (variable-based ap-maxRPC) A value $v_{i} \in D\left(x_{i}\right)$ is variablebased ap-maxRPC (or apx-maxRPC) if and only if it is value-based $p\left(x_{i}\right)$ maxRPC. A constraint network is apx-maxRPC iff all values in all domains in $D$ are apx-maxRPC. 


\subsection{Experimental evaluation of $a p$-maxRPC}

In Section 3.2 we have shown that maintaining a static form of $p$-maxRPC during the entire search can lead to a promising trade-off between computational effort and pruning when all algorithms follow the same static variable ordering. In this section, we want to put our contributions in the real context of a solver using the best known variable ordering heuristic, dom/wdeg, though it is known that this heuristic is so good that it substantially reduces the differences in performance that other features of the solver could provide. We have compared the two variants of adaptive parameterized consistency, namely apc-maxRPC and apxmaxRPC, to AC and maxRPC. We ran the four algorithms on instances of radio link frequency assignment problems, geom problems, and queens knights problems.

Table 2 reports some representative results. A first observation is that, thanks to the $d o m / w d e g$ heuristic, we were able to solve more instances before the cutoff of one hour, especially the scen11 variants of RLFAP. A second observation is that $a p c$-maxRPC and $a p x$-maxRPC are both faster than at least one of the two extreme consistencies ( $\mathrm{AC}$ and maxRPC) on all instances except scen7-w1-f4 and geo50-20-d4-75-30. Third, when apx-maxRPC and/or apcmaxRPC are faster than both AC and maxRPC (scen1-f9, scen2-f25, scen11-f9, scen11-f10 and scen11-f11), we observe that the gap in performance in terms of nodes and CCKs between AC and maxRPC is significant. Except for scen7$\mathrm{w} 1-\mathrm{f} 4$, the number of nodes visited by $\mathrm{AC}$ is three to five times greater than the number of nodes visited by maxRPC and the number of constraint checks performed by maxRPC is twelve to sixteen times greater than the number of constraint checks performed by AC. For the geom instances the CPU time of the ap-maxRPC algorithms is between $\mathrm{AC}$ and maxRPC, and it is never lower than the CPU time of AC. This probably means that when solving these instances with the dom/wdeg heuristic, there is no need for sophisticated local consistencies. In general we see that the $a p$-maxRPC algorithms fail to improve both the two extreme consistencies simultaneously for the instances where the performance gap between $\mathrm{AC}$ and maxRPC is low.

If we compare $a p x$-maxRPC to $a p c$-maxRPC, we observe that although apxmaxRPC is coarser in its design than apc-maxRPC, apx-maxRPC is often faster than apc-maxRPC. We can explain this by the fact that the constraints initially all have the same weight equal to 1 . Hence, all local parameters $a p\left(c_{k}\right)$ initially have the same value 0 , so that apc-maxRPC starts resolution by applying AC everywhere. It will start enforcing some amount of maxRPC only after the first wipe-out occurred. On the contrary, in apx-maxRPC, when constraints all have the same weight, the local parameter $p\left(x_{i}\right)$ is correlated to the degree of the variable $x_{i}$. As a result, apx-maxRPC benefits from the filtering power of $\operatorname{maxRPC}$ even before the first wipe-out.

In Table 2, we reported only the results on a few representative instances. Table 3 summarizes the entire set of experiments. It shows the average CPU time for each algorithm on all instances of the different classes of problems tested. We considered only the instances solved before the cutoff of one hour 
Table 2: Performance (CPU time, nodes and constraint checks) of AC, variable-based ap-maxRPC ( $a p x$-maxRPC), constraint-based ap-maxRPC ( apcmaxRPC), and maxRPC on various instances.

\begin{tabular}{|c|c|c|c|c|c|}
\hline & & $\mathrm{AC}$ & $a p x-\max \mathbf{R P C}$ & $a p c-\max \mathbf{R P C}$ & $\operatorname{maxRPC}$ \\
\hline \multirow[t]{3}{*}{ scen1-f9 } & $\mathrm{CPU}$ & 90.34 & 31.17 & 33.40 & 41.56 \\
\hline & \#nodes & 2,291 & 1,080 & 1,241 & 726 \\
\hline & \#ccks & $3,740,502$ & $3,567,369$ & $2,340,417$ & $50,045,838$ \\
\hline \multirow[t]{3}{*}{ scen2-f25 } & $\mathrm{CPU}$ & 70.57 & 46.40 & 27.22 & 81.40 \\
\hline & \#nodes & 12,591 & 4,688 & 3,928 & 3,002 \\
\hline & \#ccks & $15,116,992$ & $38,239,829$ & $8,796,638$ & $194,909,585$ \\
\hline \multirow[t]{3}{*}{ scen6-w2 } & $\mathrm{CPU}$ & 7.30 & 1.25 & 2.63 & 0.01 \\
\hline & \#nodes & 2,045 & 249 & 610 & 0 \\
\hline & \#ccks & $2,401,057$ & $1,708,812$ & $1,914,113$ & 85,769 \\
\hline \multirow[t]{3}{*}{ scen7-w1-f4 } & $\mathrm{CPU}$ & 0.28 & 0.17 & 0.54 & 0.30 \\
\hline & \#nodes & 567 & 430 & 523 & 424 \\
\hline & \#ccks & 608,040 & 623,258 & 584,308 & $1,345,473$ \\
\hline \multirow[t]{3}{*}{ scen11-f9 } & CPU & $2,718.65$ & $1,110.80$ & $1,552.20$ & $2,005.61$ \\
\hline & \#nodes & 103,506 & 40,413 & 61,292 & 32,882 \\
\hline & \#ccks & $227,751,301$ & $399,396,873$ & $123,984,968$ & $3,637,652,122$ \\
\hline \multirow[t]{3}{*}{ scen11-f10 } & $\mathrm{CPU}$ & 225.29 & 83.89 & 134.46 & 112.18 \\
\hline & \#nodes & 9,511 & 3,510 & 4,642 & 2,298 \\
\hline & \#ccks & $12,972,427$ & $17,778,458$ & $6,717,485$ & $156,005,235$ \\
\hline \multirow[t]{3}{*}{ scen11-f11 } & $\mathrm{CPU}$ & 156.76 & 39.39 & 93.69 & 76.95 \\
\hline & \#nodes & 7,050 & 2,154 & 3,431 & 1,337 \\
\hline & \#ccks & $7,840,552$ & $10,006,821$ & $5,143,592$ & $91,518,348$ \\
\hline \multirow[t]{3}{*}{ scen11-f12 } & $\mathrm{CPU}$ & 139.91 & 69.50 & 88.76 & 61.92 \\
\hline & \#nodes & 7,050 & 2,597 & 3,424 & 1,337 \\
\hline & \#ccks & $7,827,974$ & $11,327,536$ & $5,144,835$ & $91,288,023$ \\
\hline \multirow{3}{*}{ geo50-20d4-75-19 } & $\overline{\mathrm{CPU}}$ & 242.13 & 553.53 & 657.72 & 982.34 \\
\hline & \#nodes & 195,058 & 114,065 & 160,826 & 71,896 \\
\hline & \#ccks & $224,671,319$ & $594,514,132$ & $507,131,322$ & $2,669,750,690$ \\
\hline \multirow[t]{3}{*}{ geo50-20d4-75-30 } & CPU & 0.84 & 1.01 & 1.07 & 1.02 \\
\hline & \#nodes & 359 & 115 & 278 & 98 \\
\hline & \#ccks & 261,029 & 432,705 & 313,168 & $1,880,927$ \\
\hline \multirow[t]{3}{*}{ geo50-20d4-75-84 } & $\mathrm{CPU}$ & 0.02 & 0.09 & 0.05 & 0.29 \\
\hline & \#nodes & 59 & 54 & 59 & 52 \\
\hline & \#ccks & 33,876 & 80,626 & 32,878 & 697,706 \\
\hline \multirow[t]{3}{*}{ queensK20-5-mul } & $\overline{\mathrm{CPU}}$ & 787.35 & $2,345.43$ & 709.45 & $>3600$ \\
\hline & \#nodes & 55,596 & 40,606 & 41,743 & - \\
\hline & \#ccks & $347,596,389$ & $6,875,941,876$ & $379,826,516$ & - \\
\hline \multirow[t]{3}{*}{ queensK15-5-add } & CPU & 24.69 & 17.01 & 14.98 & 35.05 \\
\hline & \#nodes & 24,639 & 12,905 & 12,677 & 11,595 \\
\hline & \#ccks & $90,439,795$ & $91,562,150$ & $58,225,434$ & $394,073,525$ \\
\hline
\end{tabular}

by at least one of the four algorithms. To compute the average CPU time of an algorithm on a class of instances, we add the CPU time needed to solve each instance solved before the cutoff of one hour, and for the instances not solved before the cutoff, we add one hour. We observe that the adaptive approach is, on average, faster than the two extreme consistencies AC and maxRPC, except on the geom class.

In $a p x$-maxRPC and $a p c$-maxRPC, we update the local parameters $p\left(x_{i}\right)$ or $p\left(c_{k}\right)$ at each node in the search tree. We could wonder if such a frequent update does not produce too much overhead. To answer this question we performed a simple experiment in which we update the local parameters every 10 nodes 
Table 3: Average CPU time of AC, variable-based ap-maxRPC (apx-maxRPC), constraint-based $a p$-maxRPC (apc-maxRPC), and maxRPC on all instances of each class of problems tested, when the local parameters are updated at each node

\begin{tabular}{llrrrr}
\hline \multicolumn{2}{c}{ class (\#instances) } & AC & $a p x-$ maxRPC & $a p c-$ maxRPC & maxRPC \\
\hline geom (10) & \#solved & $\mathbf{1 0}$ & 10 & 10 & 10 \\
& average CPU & $\mathbf{6 9 . 2 8}$ & 180.57 & 191.03 & 279.30 \\
\hline scen (10) & \#solved & 10 & 10 & $\mathbf{1 0}$ & 10 \\
& average CPU & 18.95 & 9.63 & $\mathbf{8 . 3 0}$ & 13.94 \\
\hline \multirow{2}{*}{ scen11 (10) } & \#solved & 4 & $\mathbf{4}$ & 4 & 4 \\
& average CPU & 810.15 & $\mathbf{3 2 5 . 9 0}$ & 467.28 & 564.17 \\
\hline queensK (11) & \#solved & 6 & 6 & $\mathbf{6}$ & 5 \\
& average CPU & 135.95 & 395.41 & $\mathbf{1 2 1 . 7 5}$ & $>610.51$ \\
\hline
\end{tabular}

Table 4: Average CPU time of AC, variable-based ap-maxRPC (apx-maxRPC), constraint-based ap-maxRPC (apc-maxRPC), and maxRPC on all instances of each class of problems tested, when the local parameters are updated every 10 nodes

\begin{tabular}{llrrrr}
\hline class (\#instances) & AC & $a p x-$ maxRPC & $a p c-$ maxRPC & maxRPC \\
\hline geom (10) & \#solved & $\mathbf{1 0}$ & 10 & 10 & 10 \\
& average CPU & $\mathbf{6 9 . 2 8}$ & 147.20 & 189.42 & 279.30 \\
\hline scen (10) & \#solved & 10 & 10 & $\mathbf{1 0}$ & 10 \\
& average CPU & 18.95 & $\mathbf{7 . 4 0}$ & 8.86 & 13.94 \\
\hline \multirow{2}{*}{ scen11 (10) } & \#solved & 4 & $\mathbf{4}$ & 4 & 4 \\
& average CPU & 810.15 & $\mathbf{3 1 1 . 7 4}$ & 417.97 & 564.17 \\
\hline queensK (11) & \#solved & 6 & 6 & $\mathbf{6}$ & 5 \\
& average CPU & 135.95 & 269.51 & $\mathbf{1 1 7 . 1 8}$ & $>610.52$ \\
\hline
\end{tabular}

only. We re-ran the whole set of experiments with this new setting. Table 4 reports the average CPU time for these results. We observe that when the local parameters are updated every 10 nodes, the gain for the adaptive approach is, on average, greater than when the local parameters are updated at each node. This gives room for improvement, by trying to adapt the frequency of update of these parameters.

\section{Partition-One-Arc-Consistency}

In this section, we describe our second approach, which is inspired from singleton-based consistencies. Singleton Arc Consistency (SAC) [DB97] makes a singleton test by enforcing arc consistency and can only prune values in the variable domain on which it currently performs singleton tests. Partition-One-AC (POAC) [BA01] is an extension of SAC, which, as observed in [BD08], combines singleton tests and constructive disjunction [VSD98]. POAC can prune values everywhere in the network as soon as a variable has been completely singleton tested.

We propose an adaptive version of POAC, where the number of times vari- 
ables are processed for singleton tests on their values is dynamically and automatically adapted during search. Before moving to adaptive partition-one-AC, we first propose an efficient algorithm enforcing POAC and we compare its behaviour to SAC.

\subsection{The algorithm}

The efficiency of our POAC algorithm, POAC1, is based on the use of counters associated with each value $\left(x_{j}, v_{j}\right)$ in the constraint network. These counters are used to count how many times a value $v_{j}$ from a variable $x_{j}$ is pruned during the sequence of POAC tests on all the values of another variable $x_{i}$ (the varPOAC call to $x_{i}$ ). If $v_{j}$ is pruned $\left|D\left(x_{i}\right)\right|$ times, this means that it is not POAC and can be removed from $D\left(x_{j}\right)$.

POAC1 (Algorithm 4) starts by enforcing arc consistency on the network (line $2)$. Then it puts all variables in the ordered cyclic list $S$ using any total ordering on $X$ (line 3). varPOAC iterates on all variables from $S$ (line 7 ) to make them POAC until the fixpoint is reached (line 12) or a domain wipe-out occurs (line 8). The counter FPP (FixPoint Proof) counts how many calls to varPOAC have been processed in a row without any change in any domain (line 9).

The procedure varPOAC (Algorithm 5 ) is called to establish POAC w.r.t. a variable $x_{i}$. It works in two steps. The first step enforces arc consistency in each sub-network $N=\left(X, D, C \cup\left\{x_{i}=v_{i}\right\}\right)$ (line 4) and removes $v_{i}$ from $D\left(x_{i}\right)$ (line 5) if the sub-network is arc-inconsistent. Otherwise, the procedure TestAC (Algorithm 6) increments the counter associated with every arc inconsistent value $\left(x_{j}, v_{j}\right), j \neq i$ in the sub-network $N=\left(X, D, C \cup\left\{x_{i}=v_{i}\right\}\right)$. (Lines 6 and 7 have been added for improving the performance in practice but are not necessary for reaching the required level of consistency.) In line 8 the Boolean CHANGE is set to true if $D\left(x_{i}\right)$ has changed. The second step deletes all the values $\left(x_{j}, v_{j}\right), j \neq i$ with a counter equal to $\left|D\left(x_{i}\right)\right|$ and sets back the counter of each value to 0 (lines 12-13). Whenever a domain change occurs in $D\left(x_{j}\right)$, if the domain is empty, varPOAC returns failure (line 14); otherwise it sets the Boolean CHANGE to true (line 15).

Enforcing arc consistency on the sub-networks $N=\left(X, D, C \cup\left\{x_{i}=v_{i}\right\}\right)$ is done by calling the procedure TestaC (Algorithm 6). TestAC just checks whether arc consistency on the sub-network $N=\left(X, D, C \cup\left\{x_{i}=v_{i}\right\}\right)$ leads to a domain wipe-out or not. It is an instrumented $\mathrm{AC}$ algorithm that increments a counter for all removed values and restores them all at the end. In addition to the standard propagation queue $Q$, TestaC uses a list $L$ to store all the removed values. After the initialisation of $Q$ and $L$ (lines 2-3), TestAC revises each $\operatorname{arc}\left(x_{j}, c_{k}\right)$ in $Q$ and adds each removed value $\left(x_{j}, v_{j}\right)$ to $L$ (lines 5-10). If a domain wipe-out occurs (line 11), TestAC restores all removed values (line 12) without incrementing the counters (call to RestoreDomains with UPDATE = false) and it returns failure (line 13). Otherwise, if values have been pruned from the revised variable (line 14) it puts in $Q$ the neighbouring arcs to be revised. At the end, removed values are restored (line 16) and their counters are incremented (call to RestoreDomains with UPDATE $=$ true) before returning 


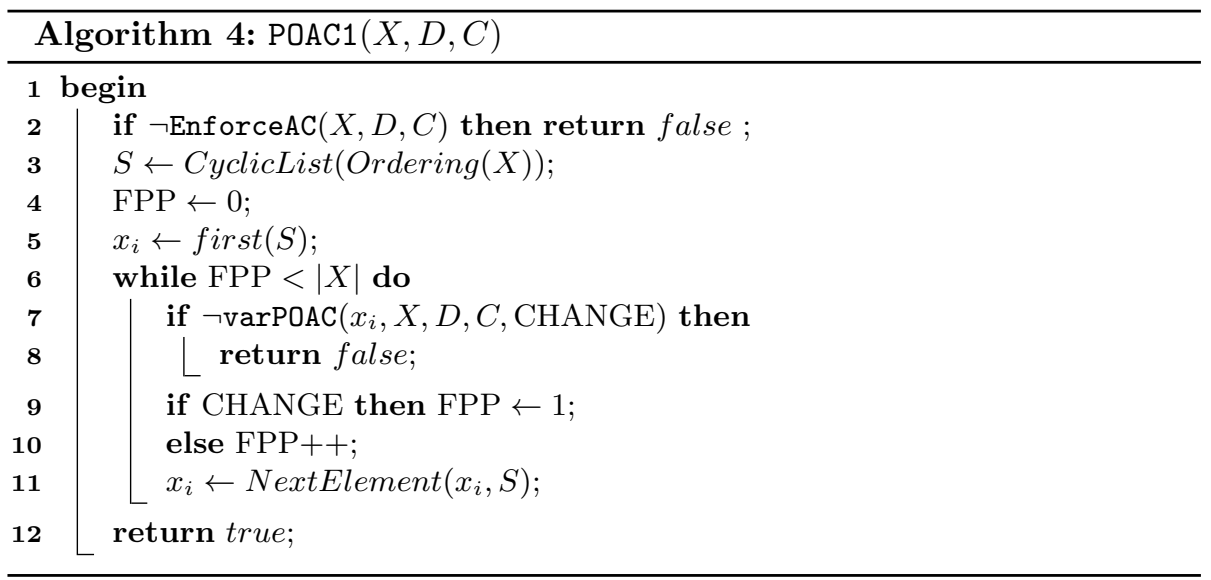

success (line 17).

Proposition 1 POAC1 has a worst-case time complexity in $O\left(n^{2} d^{2}(T+n)\right)$, where $T$ is the time complexity of the arc-consistency algorithm used for singleton tests, $n$ is the number of variables, and $d$ is the number of values in the largest domain.

Proof. The cost of calling varPOAC on a single variable is $O(d T+n d)$ because varPOAC runs $\mathrm{AC}$ on $d$ values and updates $n d$ counters. In the worst case, each of the $n d$ value removals trigger $n$ calls to varPOAC. Therefore POAC1 has a time complexity in $O\left(n^{2} d^{2}(T+n)\right)$.

\subsection{Comparison of POAC and SAC behaviors}

Although POAC has a worst-case time complexity greater than SAC, we observed in practice that maintaining POAC during search is often faster than maintaining SAC. This behavior occurs even when POAC cannot remove more values than $\mathrm{SAC}$, i.e. when the same number of nodes is visited with the same static variable ordering. This is due to what we call the (filtering) convergence speed: when both $\mathrm{POAC}$ and $\mathrm{SAC}$ reach the same fixpoint, POAC reaches the fixpoint with fewer singleton tests than SAC.

Figure 4 compares the convergence speed of POAC and SAC on an CSP instance where they have the same fixpoint. We observe that POAC is able to reduce the domains, to reach the fixpoint, and to prove the fixpoint, all in fewer singleton tests than SAC. This pattern has been observed on most of the instances and whatever ordering was used in the list $S$. The reason is that each time POAC applies varPOAC to a variable $x_{i}$, it is able to remove inconsistent values from $D\left(x_{i}\right)$ (like $\mathrm{SAC}$ ), but also from any other variable domain (unlike $\mathrm{SAC})$. 


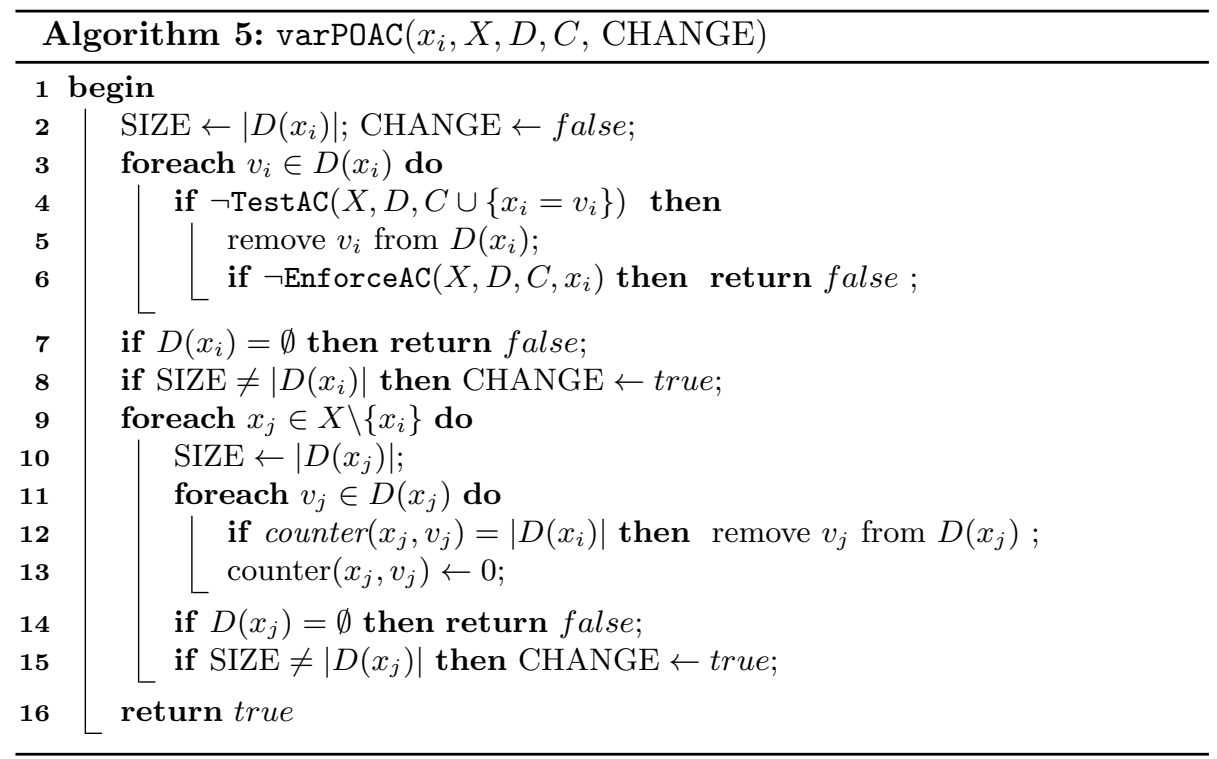

The fact that SAC cannot remove values in variables other than the one on which the singleton test is performed makes it a poor candidate for adapting the number of singleton tests. A SAC-inconsistent variable/value pair never singleton tested has no chance to be pruned by such a technique.

\section{Adaptive POAC}

This section presents an adaptive version of POAC that approximates POAC by monitoring the number of variables on which to perform singleton tests.

To achieve POAC, POAC1 calls the procedure varPOAC until it has proved that the fixpoint is reached. This means that, when the fixpoint is reached, POAC1 needs to call $n$ (additional) times the procedure varPOAC without any pruning to prove that the fixpoint was reached. Furthermore, we experimentally observed that in most cases there is a long sequence of calls to varPOAC that prune very few values, even before the fixpoint has been reached (see Figure 4 as an example). The goal of Adaptive POAC (APOAC) is to stop iterating on varPOAC as soon as possible. We want to benefit from strong propagation of singleton tests while avoiding the cost of the last calls to varPOAC that delete very few values or no value at all.

\subsection{Principle}

The APOAC approach alternates between two phases during search: a short learning phase and a longer exploitation phase. One of the two phases is executed on a sequence of nodes before switching to the other phase for another 


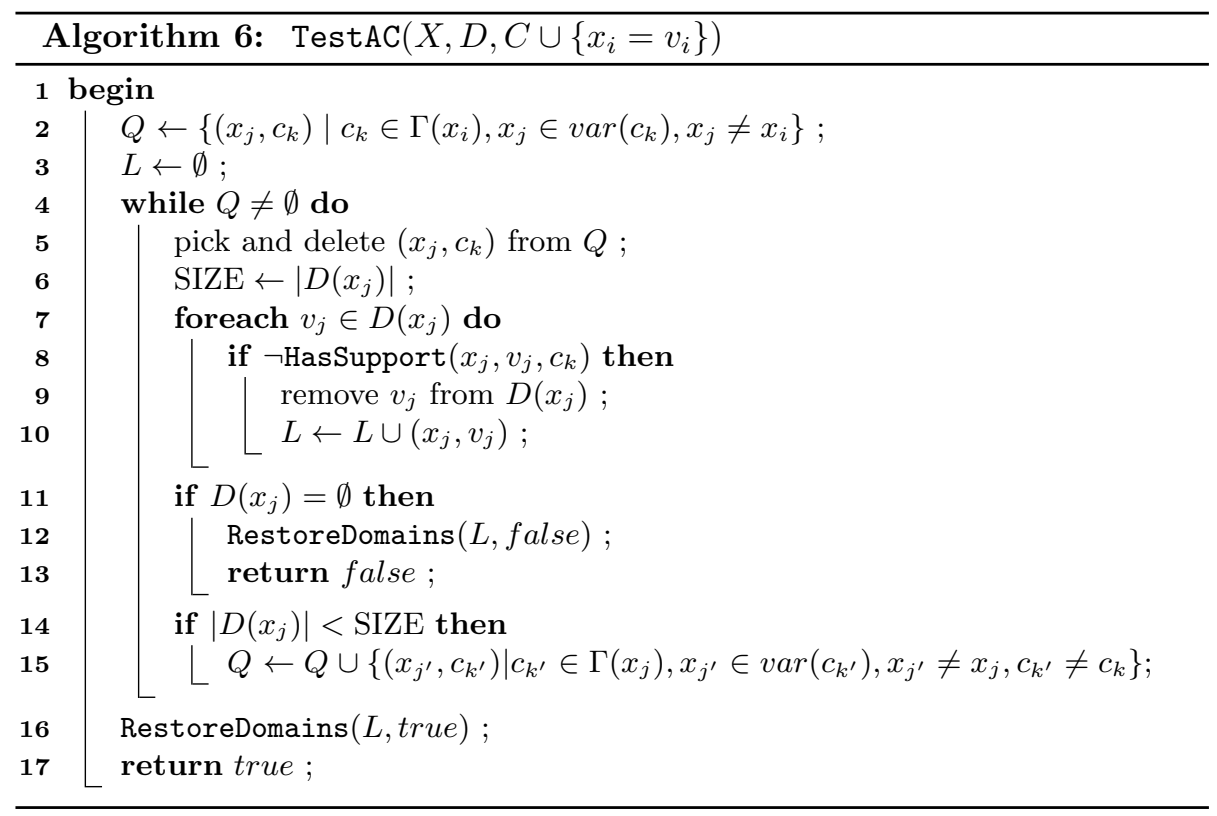

sequence of nodes. The search starts with a learning phase. The total length of a pair of sequences learning + exploitation is fixed to the parameter $L E$.

Before providing a more detailed description, let us define the ( $\log _{2}$ of the) volume of a constraint network $N=(X, D, C)$, used to approximate the size of the search space:

$$
V=\log _{2} \prod_{i=1}^{n}\left|D\left(x_{i}\right)\right|
$$

We use the logarithm of the volume instead of the volume itself, because of the large integers the volume generates. We also could have used the perimeter (i.e., $\left.\sum_{i}\left|D\left(x_{i}\right)\right|\right)$ for approximating the search space size, as done in [NT13].

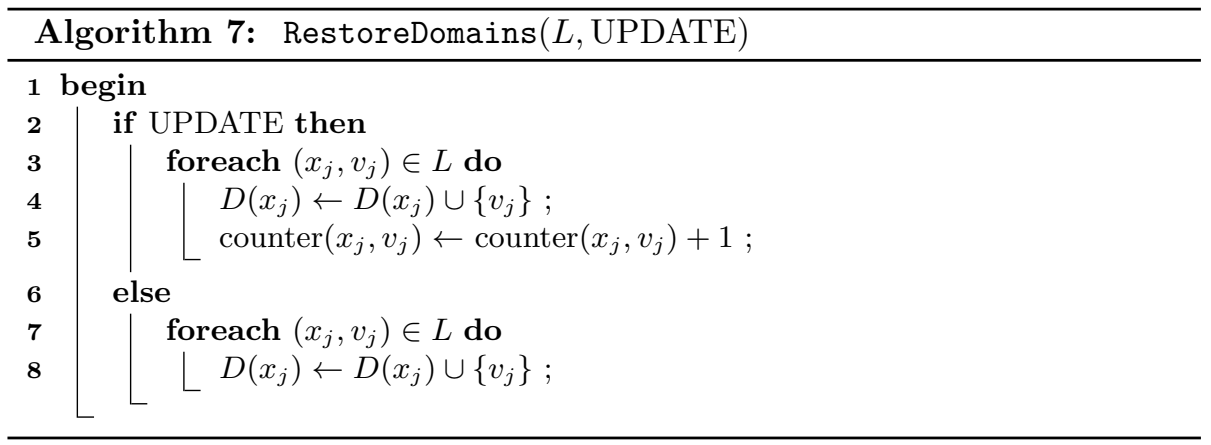




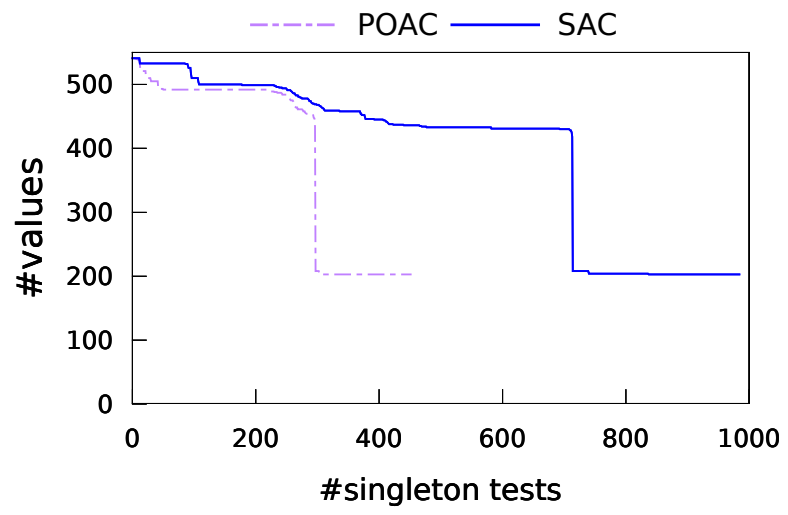

Figure 4: The convergence speed of POAC and SAC.

However, experiments have confirmed that the volume is a more precise and effective criterion for adaptive POAC.

The $i$ th learning phase is applied to a sequence of $L=\frac{1}{10} \cdot L E$ consecutive nodes. During that phase, we learn a cutoff value $k_{i}$, which is the maximum number of calls to the procedure varPOAC that each node of the next ( $i$ th) exploitation phase will be allowed to perform. A good cutoff $k_{i}$ is such that varPOAC removes many inconsistent values (that is, obtains a significant volume reduction in the network) while avoiding calls to varPOAC that delete very few values or no value at all. During the $i$ th exploitation phase, applied to a sequence of $\frac{9}{10} \cdot L E$ consecutive nodes, the procedure varPOAC is called at each node until fixpoint is proved or the cutoff limit of $k_{i}$ calls to varPOAC is reached.

The $i$ th learning phase works as follows. Let $k_{i-1}$ be the cutoff learned at the previous learning phase. We initialize $\max K$ to $\max \left(2 \cdot k_{i-1}, 2\right)$. At each node $n_{j}$ in the new learning sequence $n_{1}, n_{2}, \ldots n_{L}$, APOAC is used with a cutoff $\max K$ on the number of calls to the procedure varPOAC. APOAC stores the sequence of volumes $\left(V_{1}, \ldots, V_{\text {last }}\right)$, where $V_{p}$ is the volume resulting from the $p$ th call to varPOAC and last is the smallest among $\max K$ and the number of calls needed to prove fixpoint. Once the fixpoint is proved or the maxKth call to varPOAC performed, APOAC computes $k_{i}(j)$, the number of varPOAC calls that are enough to sufficiently reduce the volume while avoiding the extra cost of the last calls that remove few or no value. (The criteria to decide what 'sufficiently' means are described in Section 6.2.) Then, to make the learning phase more adaptive, $\max K$ is updated before starting node $n_{j+1}$. If $k_{i}(j)$ is close to $\max K$, that is, greater than $\frac{3}{4} \cdot \max K$, we increase $\max K$ by $20 \%$. If $k_{i}(j)$ is less than $\frac{1}{2} \cdot \max K$, we reduce $\max K$ by $20 \%$. Otherwise, $\max K$ is unchanged. Once the learning phase ends, APOAC computes the cutoff $k_{i}$ that will be applied to the next exploitation phase. $k_{i}$ is an aggregation of the $k_{i}(j)$ values, $j=1, \ldots, L$, computed using one of the aggregation techniques presented in Section 6.3. 
Table 5: Total number of instances solved by AC, several variants of APOAC, and POAC.

\begin{tabular}{llllcccc}
\hline$k_{i}(j)$ & $k_{i}$ & & AC & APOAC-2 & APOAC-n & APOAC-fp & POAC \\
\hline \hline LR & $70-P E R$ & \#solved & 115 & 116 & $\mathbf{1 1 9}$ & 118 & 115 \\
& Med & \#solved & 115 & 114 & $\mathbf{1 1 8}$ & $\mathbf{1 1 8}$ & 115 \\
\hline \hline LD & $70-P E R$ & \#solved & 115 & 117 & $\mathbf{1 2 1}$ & 120 & 115 \\
& Med & \#solved & 115 & 116 & $\mathbf{1 1 9}$ & $\mathbf{1 1 9}$ & 115 \\
\hline
\end{tabular}

\subsection{Computing $k_{i}(j)$}

We implemented APOAC using two different techniques to compute $k_{i}(j)$ at a node $n_{j}$ of the learning phase:

- LR (Last Reduction) $k_{i}(j)$ is the rank of the last call to varPOAC that reduced the volume of the constraint network.

- LD (Last Drop) $k_{i}(j)$ is the rank of the last call to varPOAC that has produced a significant drop of the volume. The significance of a drop is captured by a ratio $\beta \in[0,1]$. More formally, $k_{i}(j)=\max \left\{p \mid V_{p} \leq\right.$ $\left.(1-\beta) V_{p-1}\right\}$.

\subsection{Aggregation of the $k_{i}(j)$ values}

Once the $i$ th learning phase is complete, APOAC aggregates the $k_{i}(j)$ values computed during that phase to generate $k_{i}$, the new cutoff value on the number of calls to the procedure varPOAC allowed at each node of the $i$ th exploitation phase. We propose two techniques to aggregate the $k_{i}(j)$ values into $k_{i}$.

- Med $k_{i}$ is the median of the $k_{i}(j), j \in 1 . . L$.

- $q$-PER This technique generalizes the previous one. Instead of taking the median, we use any percentile. That is, $k_{i}$ is equal to the smallest value among $k_{i}(1), \ldots, k_{i}(L)$ such that $q \%$ of the values among $k_{i}(1), \ldots, k_{i}(L)$ are less than or equal to $k_{i}$.

Several variants of APOAC can be proposed, depending on how we compute the $k_{i}(j)$ values in the learning phase and how we aggregate the different $k_{i}(j)$ values. In the next section, we give an experimental comparison of the different variants we tested.

\subsection{Experimental evaluation of (A)POAC}

This section presents experiments that compare the performance of maintaining $\mathrm{AC}, \mathrm{POAC}$, or adaptive variants of POAC during search. For the adaptive variants we use two techniques to determine $k_{i}(j)$ : the last reduction (LR) and the last drop (LD) with $\beta=5 \%$ (see Section 6.2). We also use two techniques to 
Table 6: CPU time for AC, APOAC-2, APOAC-n, APOAC-fp and POAC on the eight problem classes.

\begin{tabular}{|c|c|c|c|c|c|c|}
\hline \multirow{3}{*}{$\begin{array}{l}\text { class (\#instances) } \\
\text { Tsp-20 (15) }\end{array}$} & & $\mathbf{A C}$ & APOAC-2 & APOAC-n & APOAC-fp & POAC \\
\hline & \#solved & 15 & 15 & 15 & 15 & 15 \\
\hline & sum CPU & $1,596.38$ & $3,215.07$ & $4,830.10$ & $7,768.33$ & $18,878.81$ \\
\hline \multirow[t]{2}{*}{ Tsp-25 (15) } & \#solved & 15 & 14 & 15 & 15 & 11 \\
\hline & sum CPU & $20,260.08$ & $>37,160.63$ & $16,408.35$ & $33,546.10$ & $>100,947.01$ \\
\hline \multirow[t]{2}{*}{ renault $(50)$} & \#solved & 50 & 50 & 50 & 50 & 50 \\
\hline & sum CPU & 837.72 & $2,885.66$ & $11,488.61$ & $15,673.81$ & $18,660.01$ \\
\hline \multirow[t]{2}{*}{ cril $(8)$} & \#solved & 4 & 5 & 7 & 7 & 7 \\
\hline & sum CPU & $>45,332.55^{4}$ & $>42,436.17$ & 747.05 & 876.57 & $1,882.88$ \\
\hline \multirow[t]{2}{*}{ mug (8) } & \#solved & 5 & 6 & 6 & 6 & 6 \\
\hline & sum CPU & $>29,931.45$ & $12,267.39$ & $12,491.38$ & $12,475.66$ & $2,758.10$ \\
\hline \multirow[t]{2}{*}{ K-insertions (10) } & \#solved & 4 & 5 & 6 & 5 & 5 \\
\hline & sum CPU & $>30,614.45$ & $>29,229.71$ & $27,775.40$ & $>29,839.39$ & $>20,790.69$ \\
\hline \multirow[t]{2}{*}{ myciel (15) } & \#solved & 12 & 12 & 12 & 12 & 11 \\
\hline & sum CPU & $1,737.12$ & $2,490.15$ & $2,688.80$ & $2,695.32$ & $>20,399.70$ \\
\hline \multirow[t]{2}{*}{ Qwh-20 (10) } & \#solved & 10 & 10 & 10 & 10 & 10 \\
\hline & sum CPU & $16,489.63$ & $12,588.54$ & $11,791.27$ & $12,333.89$ & $27,033.73$ \\
\hline \multicolumn{2}{|c|}{ Sum of CPU times } & $>146,799$ & $>142,273$ & 88,221 & $>115,209$ & $>211,351$ \\
\hline \multicolumn{2}{|c|}{ Sum of average CPU times per class } & $>18,484$ & $>14,717$ & 8,773 & $>9,467$ & $>10,229$ \\
\hline
\end{tabular}

aggregate these $k_{i}(j)$ values: the median (Med) and the $q$ th percentile ( $q$-PER) with $q=70 \%$ (see Section 6.3). In experiments not presented in this paper we tested the performance of APOAC using using the 10th to 90th percentiles. The 70th percentile showed the best behavior. We have performed experiments for the four variants obtained by combining two by two the parameters LR vs LD and Med vs 70-PER. For each variant we compared three initial values for the $\max K$ used by the first learning phase: $\max K \in\{2, n, \infty\}$, where $n$ is the number of variable in the instance to be solved. These three versions are denoted by APOAC-2, APOAC-n and APOAC-fp respectively.

We compare these search algorithms on instances available from Lecoutre's webpage. $^{2}$ We selected four binary classes containing at least one difficult instance for MAC (> 10 seconds): mug, K-insertions, myciel and Qwh-20. We also selected all the n-ary classes in extension: the traveling-salesman problem (TSP-20, TSP-25), the Renault Megane configuration problem (Renault) and the Cril instances (Cril). These eight problem classes contain instances with 11 to 1406 variables, domains of size 3 to 1600 and 20 to 9695 constraints.

For the search algorithm maintaining AC, the algorithm AC2001 (resp. GAC2001) [BRYZ05] is used for the binary (resp. non-binary) problems. The same AC algorithms are used as refutation procedure for POAC and APOAC algorithms. The dom/wdeg heuristic [BHLS04] is used both to order variables in the Ordering $(X)$ function (see line 3 of Algorithm 4) and to order variables during search for all the search algorithms. The results presented involve all the instances solved before the cutoff of 15,000 seconds by at least one algorithm.

Table 5 compares all the competitors and shows the number of instances

\footnotetext{
${ }^{2}$ www.cril.univ-artois.fr/ lecoutre/benchmarks.html
} 


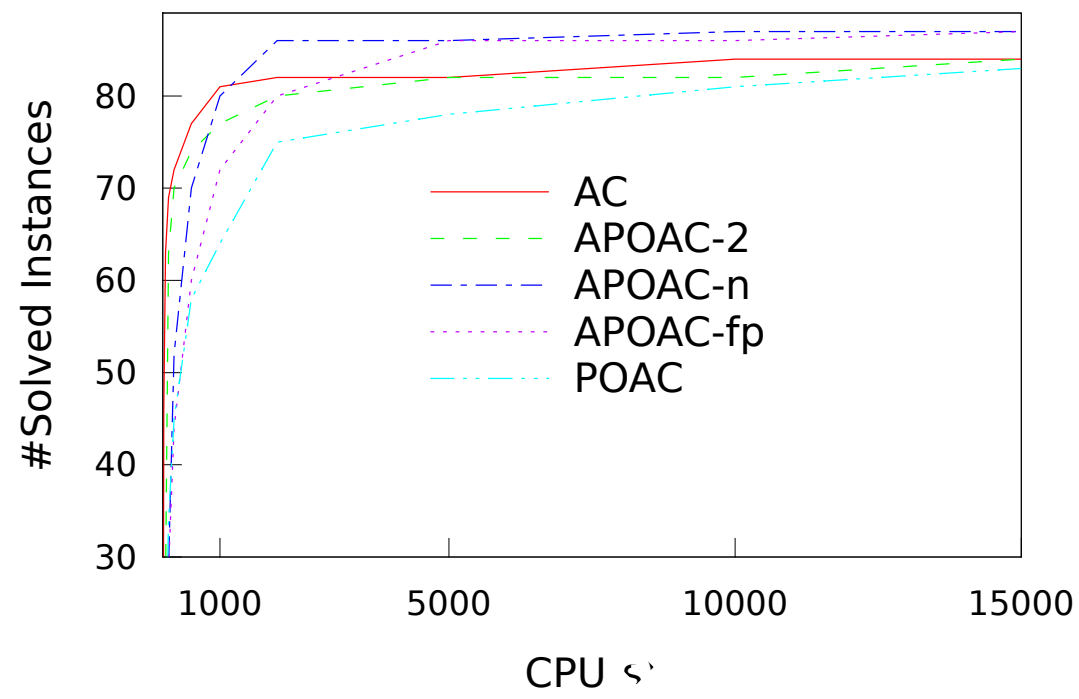

Figure 5: Number of instances solved when the time allowed increases.

(\#solved) solved before the cutoff. We observe that, on the set of instances tested, adaptive versions of POAC are better than AC and POAC. All of them, except APOAC-2+LR+Med, solve more instances than AC and POAC. All the versions using the last drop (LD) technique to determine the $k_{i}(j)$ values in the learning phase are better than those using last reduction (LR). We also see that the versions that use the 70th percentile (70-PER) to aggregate the $k_{i}(j)$ values are better than those using the median (Med). This suggests that the best combination is LD+70-PER. This is the only combination we will consider in the following.

Table 6 focuses on the performance of the three variants of APOAC (APOAC-2, APOAC-n and APOAC-fp), all with the combination (LD+70PER). The second column reports the number \#SolvedbyOne of instances solved before the cutoff by at least one algorithm. For each algorithm and each class, Table 6 shows the sum of CPU times required to solve those \#SolvedbyOne instances. When a competitor cannot solve an instance before the cutoff, we count 15,000 seconds for that instance and we write ' $>$ ' in front of the corresponding sum of CPU times. The last two rows of the table give the sum of $\mathrm{CPU}$ times and the sum of average CPU times per class. For each class taken separately, the three versions of APOAC are never worse than AC and POAC at the same time. APOAC-n solves all the instances solved by AC and POAC, and for four of the eight problem classes it outperforms both AC and POAC. However, there remain a few classes, such as Tsp-20 and renault, where even the first learning phase of APOAC is too costly to compete with AC despite our agile auto-adaptation policy that limits the number of calls to varPOAC during learning (see Section 6.1). Table 6 also shows that maintaining a high level of 
Table 7: Performance of APOAC-n compared to AC and POAC on n-ary problems.

\begin{tabular}{cccc}
\hline & AC & APOAC-n & POAC \\
\hline \#solved & $84 / 87$ & $\mathbf{8 7} / \mathbf{8 7}$ & $83 / 87$ \\
\hline sum CPU & $>68,027$ & $\mathbf{3 3 , 4 7 4}$ & $>140,369$ \\
\hline \hline gain w.r.t. $\boldsymbol{A C}$ & - & $>51 \%$ & - \\
\hline gain $\boldsymbol{w}$.r.t. $\boldsymbol{P O A C}$ & - & $>76 \%$ & - \\
\hline
\end{tabular}

consistency, such as POAC, throughout the entire network generally produces a significant overhead.

Table 7 and Figure 5 sum up the performance results obtained on all the instances with n-ary constraints. The binary classes are not included in the table and figure, because they have not been exhaustively tested. Figure 5 gives the performance profile for each algorithm presented in Table 6: AC, APOAC-2, APOAC-n, APOAC-fp and POAC. Each point $(t, i)$ on a curve indicates the number $i$ of instances that an algorithm can solve in less than $t$ seconds. The performance profile underlines that $\mathrm{AC}$ and APOAC are better than POAC: whatever the time given, they solve more instances than POAC. The comparison between $\mathrm{AC}$ and $\mathrm{APOAC}$ highlights two phases: A first phase (for easy instances), during which $\mathrm{AC}$ is better than APOAC, and a second phase, where APOAC becomes better than AC. Among the adaptive versions, APOAC-n is the variant with the shortest first phase (it adapts quite well to easy instances), and it remains the best even when time increases.

Finally, Table 7 compares the best APOAC version (APOAC-n) to AC and POAC on n-ary problems. The first row of the table gives the number of solved instances by each algorithm before the cutoff. We observe that APOAC-n solves more instances than AC and POAC. The second row of the table gives the sum of CPU time required to solve all the instances. Again, when an instance cannot be solved before the cutoff of 15,000 seconds, we count 15,000 seconds for that instance. We observe that APOAC-n significantly outperforms both AC and POAC. The last two rows of the table give the gain of APOAC-n w.r.t. AC and w.r.t. POAC. We see that APOAC-n has a positive total gain greater than $51 \%$ compared to AC and greater than $76 \%$ compared to POAC.

\section{Conclusion}

We have proposed two approaches to adjust the level of consistency automatically during search. For the parameterized local consistency approach, we introduced the notion of stability of values for arc consistency, a notion based on the depth of their supports in their respective domain. This approach us allows us to define levels of local consistency of increasing strength between arc consistency and a given strong local consistency. We have introduced two techniques which allow us to make the parameter adaptable dynamically and 
locally during search. As a second approach, we proposed POAC1, an algorithm that enforces partition-one-AC efficiently in practice. We have also proposed an adaptive version of POAC that monitors the number of variables on which to perform singleton tests. Our experiments show that in both approaches, adapting the level of local consistency during search can outperform both MAC and maintaining a chosen local consistency stronger than AC.

Our approaches concentrate on adapting the level of consistency between the standard arc consistency and a chosen higher level. There are many constraints (especially global constraints) on which arc consistency is already a (too) high level of consistency and on which the standard consistency is bound consistency or some simple propagation rules. In these cases, an approach to that chosen in this paper could allow us to adapt automatically between arc consistency and the given lower level.

\section{References}

[BA01] H. Bennaceur and M.-S. Affane. Partition-k-AC: An Efficient Filtering Technique Combining Domain Partition and Arc Consistency. In Proceedings of the Seventh International Conference on Principles and Practice of Constraint Programming (CP'01), LNCS 2239, Springer-Verlag, pages 560-564, Paphos, Cyprus, 2001.

[BBBT14] A. Balafrej, C. Bessiere, E.H. Bouyakhf, and G. Trombettoni. Adaptive singleton-based consistencies. In Proceedings of the TwentyEighth AAAI Conference on Artificial Intelligence (AAAI'14), pages 2601-2607, Quebec City, Canada, 2014.

[BBCB13] A. Balafrej, C. Bessiere, R. Coletta, and E.H. Bouyakhf. Adaptive parameterized consistency. In Proceedings of the Eighteenth International Conference on Principles and Practice of Constraint Programming (CP'13), LNCS 8124, Springer-Verlag, pages 143-158, Uppsala, Sweden, 2013.

[BCDL11] C. Bessiere, S. Cardon, R. Debruyne, and C. Lecoutre. Efficient algorithms for singleton arc consistency. Constraints, 16(1):25-53, 2011.

[BD08] C. Bessiere and R. Debruyne. Theoretical analysis of singleton arc consistency and its extensions. Artif. Intell., 172(1):29-41, 2008.

[Bes06] C. Bessiere. Constraint propagation. In F. Rossi, P. van Beek, and T. Walsh, editors, Handbook of Constraint Programming, chapter 3. Elsevier, 2006.

[BHLS04] F. Boussemart, F. Hemery, C. Lecoutre, and L. Sais. Boosting systematic search by weighting constraints. In Proceedings of the Sixteenth European Conference on Artificial Intelligence (ECAI'04), pages 146-150, Valencia, Spain, 2004. 
[BPSW11] T. Balafoutis, A. Paparrizou, K. Stergiou, and T. Walsh. New algorithms for max restricted path consistency. Constraints, 16(4):372406, 2011.

[BRYZ05] C. Bessiere, J. C. Régin, R. H. C. Yap, and Y. Zhang. An Optimal Coarse-grained Arc Consistency Algorithm. Artif. Intell., 165(2):165-185, 2005.

[BSW08] C. Bessiere, K. Stergiou, and T. Walsh. Domain filtering consistencies for non-binary constraints. Artif. Intell., 172(6-7):800-822, 2008.

[DB97] R. Debruyne and C. Bessiere. Some Practicable Filtering Techniques for the Constraint Satisfaction Problem. In Proceedings of the Fifteenth International Joint Conference on Artificial Intelligence (IJCAI'97), pages 412-417, Nagoya, Japan, 1997.

[DB01] R. Debruyne and C. Bessiere. Domain filtering consistencies. Journal of Artificial Intelligence Research, 14:205-230, 2001.

[KVH06] I. Katriel and P. Van Hentenryck. Randomized filtering algorithms. Technical Report CS-06-09, Brown University, June 2006.

[NT13] B. Neveu and G. Trombettoni. Adaptive Constructive Interval Disjunction. In Proceedings of the Twenty-Fifth IEEE International Conference on Tools for Artificial Intelligence (IEEE-ICTAI'13), pages 900-906, Washington D.C., USA, 2013.

[PS12] A. Paparrizou and K. Stergiou. Evaluating simple fully automated heuristics for adaptive constraint propagation. In Proceedings of the Twenty-Forth IEEE International Conference on Tools for Artificial Intelligence (IEEE-ICTAI'12), pages 880-885, Athens, Greece, 2012.

[Sel03] M. Sellmann. Approximated consistency for knapsack constraints. In Proceedings of the Ninth International Conference on Principles and Practice of Constraint Programming (CP'03), LNCS 2833, Springer-Verlag, pages 679-693, Kinsale, Ireland, 2003.

[SS09] E. Stamatatos and K. Stergiou. Learning how to propagate using random probing. In Proceedings of the Sixth International Conference on Integration of $A I$ and $O R$ Techniques in Constraint Programming for Combinatorial Optimization Problems (CPAIOR'09), LNCS 5547, Springer, pages 263-278, Pittsburgh PA, 2009.

[Ste08] K. Stergiou. Heuristics for dynamically adapting propagation. In Proceedings of the Eighteenth European Conference on Artificial Intelligence (ECAI'08), pages 485-489, Patras, Greece, 2008. 
[Ste09] K. Stergiou. Heuristics for dynamically adapting propagation in constraint satisfaction problems. AI Commun., 22:125-141, August 2009.

[TC07] G. Trombettoni and G. Chabert. Constructive Interval Disjunction. In Proceedings of the Thirteenth International Conference on Principles and Practice of Constraint Programming (CP'07), LNCS 4741, Springer-Verlag, pages 635-650, Providence RI, 2007.

[VSD98] P. Van Hentenryck, V. A. Saraswat, and Y. Deville. Design, implementation, and evaluation of the constraint language cc(FD). $J$. Log. Program., 37(1-3):139-164, 1998. 